Published in final edited form as:

Expert Opin Emerg Drugs. 2012 December ; 17(4): 469-492. doi:10.1517/14728214.2012.744393.

\title{
Emerging human papillomavirus vaccines
}

\author{
Barbara Ma ${ }^{1}$, Bharat Maraj ${ }^{1}$, Nam Phuong Tran ${ }^{1}$, Jayne Knoff ${ }^{1}$, Alexander Chen ${ }^{1}$, Ronald D \\ Alvarez $^{5}$, Chien-Fu Hung ${ }^{1,2}$, and T.-C. Wu ${ }^{\dagger, 1,2,3,4}$ \\ ${ }^{1}$ The Johns Hopkins Medical Institutions, Departments of Pathology, Baltimore, MD, USA \\ ${ }^{2}$ The Johns Hopkins Medical Institutions, Departments of Oncology, Baltimore, MD, USA \\ ${ }^{3}$ The Johns Hopkins Medical Institutions, Departments of Obstetrics and Gynecology, Baltimore, \\ MD, USA \\ ${ }^{4}$ The Johns Hopkins Medical Institutions, Departments of Molecular Microbiology and \\ Immunology, Baltimore, MD, USA \\ ${ }^{5}$ University of Alabama at Birmingham, Department of Obstetrics and Gynecology, Birmingham, \\ MD, USA
}

\begin{abstract}
Introduction-Identification of human papillomavirus (HPV) as the etiologic factor of cervical, anogenital, and a subset of head and neck cancers has stimulated the development of preventive and therapeutic HPV vaccines to control HPV-associated malignancies. Excitement has been generated by the commercialization of two preventive L1-based vaccines, which use HPV viruslike particles (VLPs) to generate capsid-specific neutralizing antibodies. However, factors such as high cost and requirement for cold chain have prevented widespread implementation where they are needed most.
\end{abstract}

Areas covered-Next generation preventive HPV vaccine candidates have focused on costeffective stable alternatives and generating broader protection via targeting multivalent L1 VLPs, L2 capsid protein, and chimeric L1/L2 VLPs. Therapeutic HPV vaccine candidates have focused on enhancing T cell-mediated killing of HPV-transformed tumor cells, which constitutively express HPV-encoded proteins, E6 and E7. Several therapeutic HPV vaccines are in clinical trials.

Expert opinion-Although progress is being made, cost remains an issue inhibiting the use of preventive HPV vaccines in countries that carry the majority of the cervical cancer burden. In addition, progression of therapeutic HPV vaccines through clinical trials may require combination strategies employing different therapeutic modalities. As research in the development of HPV vaccines continues, we may generate effective strategies to control HPV-associated malignancies.

\section{Keywords}

clinical trials; human papillomavirus; immunotherapy; vaccines

\footnotetext{
(C) 2012 Informa UK, Ltd.

${ }^{\dagger}$ Author for correspondence: Tel: +1 410614 3899; Fax: +1 443287 4295; wutc@jhmi.edu.

Declaration of interest

This work was supported by the NCI SPORE in Cervical Cancer P50 CA098252, NCI 1 RO1 CA114425-06, 1RO1 CA118790, 5 R21

AI085380, and RC2CA148499-2.
} 


\section{Background}

Human papillomavirus (HPV) accounts for $5.2 \%$ of all cancers worldwide and is the causative agent of cervical, anogenital, and a subset of head and neck cancers (for review, see $[1,2])$. Among these cancers, cervical cancer is the most well-known malignancy associated with HPV infections, with an estimated 49,300 diagnoses and 274,000 deaths annually [3]. Although there are more than 100 types of HPV identified [4], the International Agency for Research on Cancer designate HPV types 16, 18, 31, 33, 35, 39, 45, 51, 52, 56, 58,59 , and 66 as 'definite carcinogens' due to their oncogenic potential [5-7]. In contrast to low-risk types that are associated with benign lesions such as condyloma accuminata, highrisk HPV types are connected with the development of high-grade lesions and malignant tumors. Persistent infection with HPV has been proven to be a causative and necessary factor in the development of squamous intraepithelial lesions (SIL), also known as cervical intraepithelial lesions (CIN), and frank malignant cervical cancer [8]. The most common 'high-risk' HPV types associated with cervical cancer are HPV-16 and HPV-18, which are responsible for about $62.6 \%$ and $15.7 \%$ of cervical cancers respectively [9]. Furthermore, among all the HPV-associated cancers, the proportion attributable to HPV types 16 and 18 is $63-80 \%$ of penile cancers, $80-86 \%$ of vulva/vaginal cancers, $93 \%$ of anal cancers, and 89 - 95\% of oropharyngeal cancers (for review, see [10]). Therefore, HPV-16 and HPV-18 have been the primary focus of HPV vaccine development.

The development of an HPV vaccine requires an understanding of the molecular biology of HPV. HPV is a non-enveloped, circular, double-stranded DNA virus belonging to the Papillomaviridae family. HPV is comprised of an icosahedral capsid enclosing an approximately 8 kilobase pair genome that encodes early proteins E1, E2, E4, E5, E6, E7, and late proteins L1 and L2. HPV early gene products regulate the viral life cycle and manipulate cell machinery to replicate, transcribe, and translate viral proteins (E1 and E2), regulate early viral gene products E6 and E7 (E2), induce cytoskeleton rearrangements (E4), and cause cell-cycle deregulation (E6 and E7). The major capsid protein (L1) and minor capsid protein (L2) are involved in packaging the virion before extracellular release (for review, see [11]).

HPV can infect the basal cells of the cervical epithelium and other epithelial tissues upon microtrauma of tissues and cells [12]. However, most HPV infections are self-limiting and transient (for review, see [13]). In a productive infection of HPV, the expression of the HPV genome is correlated with the maturation of the infected cell. Immature epithelial cells in the basal layer of epithelium allow expression of the HPV early genes while transcription shifts to the late genes in terminally differentiated cells, allowing packaged virions to be released from more superficial epithelial layers. This localization of HPV results in pathological lowgrade squamous intraepithelial lesions (LSIL, also known as CIN 1). In LSIL, the HPV genome is usually in the episomal form. Of greater concern are the high-grade squamous intraepithelial lesions (HSIL, also known as CIN 2/3), which can progress to malignancies. In some HSILs, high-risk HPV types may integrate into the host genome. This integration of HPV genes causes deletion of some of the early genes (E2, E4, E5) and late genes (L1 and L2). Since E2 is a transcriptional repressor of E6 and E7 genes, loss of E2 allows for upregulation of E6 and E7 oncogenes. These E6 and E7 proteins inactivate and degrade tumor suppressors p53 and retinoblastoma $(\mathrm{Rb})$, respectively, leading to cell-cycle deregulation, genomic instability, and uncontrolled proliferation of the host cell (for review, see $[8,14,15])$. The understanding of HPV virology and its role in tumorigenesis has facilitated the development of preventive and therapeutic HPV vaccines. 


\section{Medical need}

Cervical cancer is the third leading cause of cancer deaths in women worldwide $[13,16]$. The commercialization of preventive HPV vaccines, Gardasil and Cervarix, has important implications for preventing cervical cancer. In high-resource countries, the implementation of organized gynecological screening programs with appropriate treatment of the detected pre-cancerous lesions has decreased cervical cancer incidence. The newly commercial preventive HPV vaccines may further impact the cervical cancer rates in developed countries. However, cervical cancer imposes its greatest disease burden on underserved populations in regions such as Africa, Melanesia, South-Central Asia, and South and Central America where these vaccines are less effectively implemented [17]. Despite preventive vaccines and Pap smears, the incidence of cervical cancer continues to increase in developing countries due to the high expense and complex infrastructure necessary to implement effective screening and vaccination programs. Hence, the majority of cervical cancer cases (83\%) occur in developing countries, and the burden of cervical cancer is expected to increase to $90 \%$ in developing countries by 2020 [16]. In addition, the available preventive vaccines are effective in protecting against the oncogenic HPV types 16 and 18, but the prevalence of oncogenic strains of HPV apart from types 16 and 18 vary across populations globally [18]. Thus, broader cross-protection against more oncogenic HPV types is a highly desirable trait in the second generation of HPV vaccine candidates.

There is another important limitation to current commercial preventive vaccines in that they do not have therapeutic effects (for review, see [19]). Once a patient is infected with HPV, there is currently no effective way to eradicate a persistent HPV infection before the development of pre-cancerous lesions. Globally, there is a considerable burden of established HPV infections and HPV-associated diseases that are undetected, untreated, and slowly progressing toward malignant transformation. Even with mass vaccination using a highly effective, preventive HPV vaccine, it is estimated that it will take at least 20 years before the incidence and prevalence of cervical cancer significantly decreases due to the slow rate of carcinogenesis. Thus, there is great medical need for the development of novel preventive and therapeutic vaccines for the control of HPV-associated disease.

\section{Existing treatments}

\subsection{Existing treatments of HPV-associated lesions}

Although there is no effective treatment for persistent HPV infection, there are several approaches for treating HPV-associated lesions (for review, see [20,21]). In general, the treatment for HPV-associated lesions can be classified into surgical methods and medical approaches. For LSILs (CIN 1 lesions), in which the majority of lesions spontaneously regress, most clinicians only closely observe without aggressive intervention. For HSILs, (CIN 2/3 lesions), most of the treatments rely on surgical interventions. These conservative surgical treatments include cryotherapy, loop electrosurgical excision procedure (LEEP), cone biopsy (conization), or laser conization. Cryotherapy utilizes a cryoprobe set to below $-22^{\circ} \mathrm{C}$ to freeze and destroy abnormal tissue. LEEP uses an electrical current passed through a thin wire loop to remove abnormal tissue in a knife-like fashion. Cone biopsy involves removal of a cone-shaped piece of cervical tissue for the evaluation of any abnormalities. Laser conization employs a laser beam for the dissection of affected tissue.

Treatment of frank invasive cancer often involves surgery, radiotherapy, and/or chemotherapy. Early stages of microinvasive cervical cancer may be treated by total hysterectomy (removal of uterus as well as the cervix). For Stage IBI cervical cancer patients, radical hysterectomy (removal of the uterus, cervix, parametrium, and a portion of the upper vagina) may be used as the primary management. For advanced-stage cervical 
cancers, chemotherapy/radiation therapy combinations are the standard course of management $[22,23]$. In many cases, these procedures are responsible for not inconsequential adverse effects [24]. Analyses of conservative surgical treatments for cervical dysplasia also found that conization and LEEP had similar pregnancy-related morbidities such as pre-term delivery, low birth weight, vaginal hemorrhage, and post-birth infections [24]. In addition, these treatments are associated with significant recurrence rates of up to $10 \%$ of cases, which may be caused by persistent high-risk HPV infection, particularly HPV-16 [21,25]. Hence, the efficacy of existing surgical treatments may drop due to the risk of relapse, possibly insufficient clearance of affected tissue, and potentially undesirable side effects.

Medical approaches for treatment of HPV-associated lesions can be further classified into anti-viral agents and agents that boost host immunity. Anti-viral approaches directly inhibit HPV replication and have included the use of the systemic treatment with cidofovir (for review, see [26]), and topical podophyllin [27] for the treatment of pre-cancerous lesions and/or genital warts. Agents that boost host immunity to better control established HPVassociated lesions include interferons and Toll-like receptor agonists. Interferons, particularly IFN-a, have anti-proliferative activities and have been explored for the treatment of benign HPV-related lesions (for review, see [28,29]). However, this treatment is expensive and has limited efficacy in treating high-grade HPV-associated lesions [30]. An immunomodulatory agent that has generated interest is imiquimod, a Toll-like receptor agonist that induces immune production of interferon and several inflammatory cytokines, such as IFN-a, IL-6, and TNF-a, to potentiate innate immunity [31]. It has been shown that topical application of imiquimod can lead to the activation of Langerhans cells, which subsequently migrate to local lymph nodes to activate the adaptive immune system [32]. Imiquimod has also been used clinically in vulvar intraepithelial neoplasia (VIN) patients and was shown to lead to lesion regression [33].

\subsection{Existing commercial prophylactic HPV vaccines}

The commercialization of two preventive HPV vaccines, Gardasil (Merck, NJ, USA) and Cervarix (GlaxoSmithKline, Rixensart, Belgium), represents a huge breakthrough for the prevention of HPV-associated disease [34,35] (for review, see [36-40]). The current preventive HPV vaccines use HPV L1 major capsid protein, which can form virus-like particles (VLPs) that morphologically mimic native virions and elicit antibody production by the immune system (for review see [41]) (also see Figure 1). Gardasil is a quadrivalent vaccine that includes VLPs for high-risk types HPV-16 and HPV-18, associated with cervical cancer, as well as low-risk types HPV-6 and HPV-11, which are associated with benign genital warts. Cervarix is a bivalent vaccine that contains VLPs for HPV-16 and HPV-18 only (for review, see [42]). The vaccines also include adjuvants that aim to maximize the duration and extent of immune responses for the smallest given amount of VLP, with Gardasil being enhanced with aluminum hydroxyphosphate sulfate and Cervarix boosted by the AS04 adjuvant system of aluminum hydroxide and monophosphoryl lipid A. Though both vaccines contain the classical aluminum salts that are the standard for most vaccines in use today, Cervarix is the only vaccine that contains monophosphoryl lipid A, a Toll-like receptor 4 agonist that primes the innate immune response and helps stimulate the adaptive immune responses to further enhance antibody titers [43]. Both the quadrivalent Gardasil and bivalent Cervarix have been shown to be highly efficacious in preventing CIN1-3 or adenocarcinoma in situ (AIS) in women who are not infected with the relevant HPV types before vaccination [44-47]. Gardasil has also been shown to prevent HPV type 6 and 11-associated genital warts [48,49], HPV-related vulvar and vaginal intraepithelial neoplasia grade 1-3 [50,51], and high-grade anal disease in men [52-54]. Table 1 compares Cervarix and Gardasil. 


\section{Current research goals}

\subsection{Goals for next-generation preventive HPV vaccines}

The next generation of preventive HPV vaccines must address the issues of cost, duration of efficacy, and broadening protection (for review, see [55]). Cost is the major obstacle to the implementation of the current commercial preventive HPV vaccines in resource-poor regions (for review, see [56]). Gardasil and Cervarix each require 3 doses at the price of $\$ 120$ USD per dose and \$100 USD per dose, respectively, although tiered pricing might be negotiated. Realistically, the perdose cost for developing countries needs to be lower than $\$ 5$ to make vaccination affordable [57]. There have been efforts to investigate whether women are afforded the same protection with a 2-dose regimen instead of the current 3-dose regimen for both vaccines and preliminary results suggest that the efficacy, measured by antibody responses at one month and 24 months follow vaccination with the bivalent vaccine, was comparable between a 2-dose and 3-dose regimen [58]. Currently, there are no immediate plans to change the 3-dose regimen as GSK and Merck believe that 3 doses are optimal for a high and sustained immune response and the efficacy of the 2-dose regimen against infection and disease endpoints has not yet been demonstrated [59]. In addition, vaccines require facilities and technology for storage and transport that developing countries may lack. Vaccine producers must reduce production costs and create thermostable vaccines to make preventive HPV vaccines more affordable and practical for developing countries.

Another issue with the Gardasil and Cervarix vaccines that is under scrutiny is the duration of protection. Phase II trials provide data on the longest protective span/coverage for Gardasil at 6.4 years $[60,61]$ and for Cervarix at 8.4 years $[40,62,63]$. Analyses have indicated that the duration of vaccine efficacy must last at least 15 years for cost-effective prevention of cervical cancer [36]. In a comparative immunogenicity and safety study of the bivalent Cervarix and quadrivalent Gardasil in 1,106 healthy women aged $18-45$, Cervarix induced significantly higher serum neutralizing antibody titers for HPV-16 and HPV-18 in all age strata at 7 months after vaccination [64]. In a follow-up study of these vaccinated women at month 24 , Cervarix was found to yield geometric mean titers of neutralizing antibodies that were 2.4 - 5.8-fold higher for HPV-16 and 7.7 - 7.9-fold higher for HPV-18 compared to those generated by Gardasil [65]. Although Cervarix can generate higher antibody titers for HPV types 16 and 18 as compared to Gardasil, it is unclear if antibody titers will correlate with longevity of clinical protection against HPV infection.

Furthermore, although Gardasil and Cervarix contain VLPs for HPV-16 and-18, more than a dozen other high-risk subtypes responsible for cervical cancers are virtually unprotected against. Although HPV L1 VLPs are highly immunogenic and capable of generating neutralizing antibodies in both animals and humans, they are highly type-restricted and generally do not generate cross-protection against other types of HPV. In a 4-year randomized, controlled trial of 17,622 women aged $16-26$, Gardasil was shown to provide sustained protection against low-grade cervical, vulvar, and vaginal intraepithelial neoplasias and genital warts attributable to HPV types 6,11,16, and 18 and cause substantial reduction in the burden of these diseases up to 42 months of follow-up [48]. However, vaccine efficacy against all lesions, regardless of the associated type of HPV, was $30 \%, 75 \%, 48 \%$, and $83 \%$ for cervical, vulvar, vaginal intraepithelial neoplasia, and condyloma, respectively, suggesting that Gardasil has low cross-protection for other HPV types not included in the vaccine. Follow-up studies were also performed to analyze the efficacy and cross-protection of the bivalent Cervarix [66,67]. The efficacy of Cervarix against CIN2+ and CIN3+ lesions associated with HPV-16/18 was highest in the 15 - 17 age group and declined with increasing age, supporting current recommendations of vaccination at younger ages $[66,67]$. Although Cervarix was found to be $100 \%$ effective in preventing CIN3 + lesions caused by HPV-16 and -18, the cross-protective efficacy in 
preventing CIN2+ lesions was $84.3 \%$ for HPV-31, 59.4\% for HPV-33, and was statistically insignificant for HPV-39, $-45,-52,-58$, and -68 [66]. Hence, Cervarix was also found to have cross-protection that is limited in nature. Next-generation preventive HPV vaccines must aim to generate broader protection to substantially reduce the incidence of HPVassociated disease.

\subsection{Goals for therapeutic HPV vaccines}

HPV has a predilection for infecting epithelial cells, and in early-stage lesions above the basement membrane, no HPV antigens are present in circulatory and lymphatic systems [68]. This situation makes HPV infection difficult to generate potent humoral immunity. Furthermore, the humoral immunity against HPV also is unlikely to eradicate established HPV infections via immunoglobulins because viral proteins are not exposed to an appreciable extent on the cell surface, as they would be with viruses that have enveloped proteins that accumulate on the cell surface. Thus, it has become apparent that the immunological clearance of chronic HPV infections from the body likely requires an antigen-specific $\mathrm{T}$-cell response, rather than neutralizing antibodies generated by prophylactic HPV vaccines (Figure 2). To decrease the prevalence of HPV-associated cancer and control established HPV infections, therapeutic HPV vaccines must effectively generate cell-mediated immune responses to HPV-infected cells, pre-cancerous, and cancerous lesions. Cell-mediated immunity involves the activation of CD4+ T helper cells and CD8+ T cells by antigen-presenting cells (APCs). These T cells then specifically destroy infected cells, thus clearing HPV infections. Therefore, therapeutic vaccines that induce an antigen-specific cell-mediated response have the potential to eradicate and prevent persistent infection that leads to HPV-associated cancer. Because HPV infects the mucosal surfaces, it will be desirable to develop vaccine strategies that are capable of inducing strong mucosal T-cell and/or B-cell immunity against HPV.

Quite different from the preventive HPV vaccines that target HPV L1 and/or L2, therapeutic HPV vaccines should consider targeting HPV-encoded early proteins (E1-7). HPV E6 and E7 antigens in particular are ideal targets for therapeutic HPV vaccines because they i) are constitutively expressed only in infected cells and malignant tumors but not normal cells, ii) are foreign oncoproteins which diminish the problem of immune tolerance, and iii) are unlikely to be selected against in viral progeny in attempts to evade immune response because they are essential for cell immortalization and transformation. Therefore, multiple therapeutic HPV vaccines strategies have mainly focused on eliciting HPV E6 and E7specific T-cell immune responses. Therapeutic HPV vaccines are still currently being explored in different phases of clinical trials. Table 2 summarizes the different phases of clinical trials for therapeutic HPV vaccines. The ultimate goal for therapeutic HPV vaccine is the progression through clinical trials to commercialization.

\section{Scientific rationale and competitive environment}

\subsection{Next-generation preventive HPV vaccines}

There have been several approaches for the next generation of preventive HPV vaccines that have broader protection against more high-risk types of HPV at possibly reduced costs. These strategies include targeting multivalent VLP vaccines, L1 capsomers, and L2 vaccines (see Figure 3).

5.1.1 Multivalent VLP vaccines-Existing VLP vaccine strategies generate a neutralizing antibody response specific for HPV L1 capsid protein (Gardasil and Cervarix). It is now clear that each VLP is type-restricted. Therefore, to generate broader protection, additional types of HPV must be included in vaccines. Currently, there are ongoing trials to 
compare multivalent VLP vaccines to Gardasil [69-72]. However, several parameters remain unclear. The optimal dose needed for each type of VLP in a multivalent vaccine is unclear. In addition, it is not clear whether the presence of a VLP for one type of HPV will influence the immune response generated against another type. Furthermore, it is uncertain if the duration of protection is similar for each HPV type included within the vaccine. It is also not clear whether the inclusion of more types will make vaccines more costly than the current Gardasil and Cervarix. Nevertheless, existing multivalent VLP vaccines are currently being explored, and they warrant further investigation to provide broader protection against the majority of high-risk types of HPV.

5.1.2 L1 capsomer vaccines-Commercial HPV vaccines in production are out of reach for patients most affected by cervical cancer, and $\mathrm{L} 1$ capsomer vaccines represent a potential cost-effective alternative to current L1 VLP vaccines since monovalent L2-based vaccines can be produced in E. coli. Capsomers are the basic structural components of viral capsids. Whereas HPV L1 VLP is composed of 360 copies of the L1 protein, only five L1 monomers are needed to assemble into a thermo-stable pentavalent capsomer. The ability to produce L1 capsomers in bacteria is advantageous because they are cheaper to produce than virus-like particles made in insect cells (Cervarix) and yeast cells (Gardasil). Despite a different means of production, L1 capsomer vaccines are successful in eliciting neutralizing antibodies. Capsomers produced in E. coli $[73,74]$ or expression of the L1 gene in recombinant Salmonella enterica serovar Typhimurium [75] have successfully induced protective antibodies in pre-clinical models. Scadlich et al. have further demonstrated that HPV16 L1 capsomers can be further modified to create a mutant L1 capsomer with comparable immunogenicity to the commercial L1 VLPs [76]. Innovax currently has an E. coli-derived L1 VLP HPV 16/18 vaccine in a Phase II clinical trial (NCT01355823). However, the immunogenicity of $\mathrm{L} 1$ capsomers has not been directly compared to commercial vaccines using the same adjuvant system in humans. The ability of L1 capsomers to induce a comparable neutralizing antibody response as the commercial HPV vaccines in humans will be crucial to the future of their clinical development.

5.1.3 L2 vaccines-A promising approach to overcome the type-restricted limitations of L1 VLP vaccines is the employment of the HPV L2 minor capsid protein (for review, see [77]). L2 is highly conserved among the different HPV types and can induce broader protection through cross-neutralizing antibodies. It has been shown that immunization of animals with the amino $(\mathrm{N})$-terminal peptide of $\mathrm{L} 2$ elicited neutralizing antibodies that protect against challenges with cognate papillomavirus types in vivo [78,79], crossneutralize heterologous types in vitro [80-82], and confer cross-protection in vivo [83,84]. More recently, it has been shown that oral immunization with HPV-16 L2 (N-terminal aa1-124) expressed on the surface of Lactobacillus casei in Balb/c mice generated significant neutralizing antibodies against HPV-16, as well as cross-neutralizing antibody responses against HPV-18, -45 , and -58 pseudovirions [85]. The cross-reactivity of L2 has important implications for potentially protecting against a large spectrum of not only highrisk but also low-risk HPV types. Furthermore, L2-based vaccines may represent a costeffective alternative to current L1 VLP vaccines since L2-based vaccines can be produced in E. coli as opposed to yeast (Gardasil) or insect cells (Cervarix). The reduced cost of vaccine production may increase the developing world's accessibility to preventive HPV vaccines.

Despite these advantages, one challenge posed is that L2-based vaccines suffer from poor immunogenicity as compared to L1-based vaccines as L2 generally does not elicit a high level of neutralizing antibody titers compared to L1. One strategy to enhance the immunogenicity of L2 has been to develop a HPV L2-based vaccine candidate utilizing VLPs derived from bacteriophage PP7 [86,87]. When a broadly neutralizing epitope of L2 is displayed on PP7 VLPs, it has been shown to be highly immunogenic. Mice immunized 
with a combination of L2 VLPs derived from eight HPV subtypes were strongly protected from challenge by pseudovirions of all eight subtypes [86], suggesting that vaccines using bacteriophage VLPs may be an effective methodology to generate high titers of neutralizing antibodies to protect against a range of HPV subtypes. Additional efforts to improve the immunogenicity of L2 have focused on adjuvants, such as alum in combination with a TLR4 agonist like monophosphoryl lipid A (MPL), that provide a 'danger signal' [88].

Another focus on enhancing L2-based vaccines involves further improving crossneutralization either through the generation of a synthetic consensus region of high-risk HPV L2 sequences or through the generation of polymeric L2 antigen. A concatenated multitype L2 fusion protein consisting of known cross-neutralizing epitopes derived from several divergent HPV types (L2 from HPV 1,5,6,16,18) was shown to generate highneutralizing antibody titers to heterologous HPVs and generated protection against cervicovaginal challenge with HPV-16 pseudovirions four months after immunization [88]. Multimeric L2 fusion protein combined with Cervarix or HPV-16 L1 capsomers was also shown to broaden protection against HPV infection [89]. Upon determination of the appropriate adjuvants, the multimeric L2 vaccine will be explored in clinical trials [90].

5.1.4 Chimeric VLP vaccines-The advantages of the immunogenicity of L1 vaccines and the broad cross-protection mediated by L2 can be combined in the form of chimeric L1L2 VLPs. Since L2 is less abundant and found predominantly in the interior of the viral particle, genetically fusing or chemically conjugating L2 to the L1 major capsid protein such that L2 is inserted into the L1 VLP surface may generate more immunogenic, crossprotective immune responses against multiple HPV genotypes. Such a chimeric vaccine would stimulate production of both anti-L1 and anti-L2 neutralizing antibody responses. Immunization of rabbits with chimeric VLPs with L2-peptides on their surface was shown to induce an antibody neutralizing HPV16 as well as elicit antibodies cross-neutralizing HPV18, 31, 52, and 58 pseudovirions [91]. It has also been recently shown that a type of chimeric VLP, in which HPV16 VLP was combined with the type-common L2-epitope, induced in rabbits a high level of the anti-HPV16 L1 neutralizing antibody and a lower but sufficient level of the anti-L2 cross-neutralizing antibody to be protective [92]. Furthermore, vaccination with chimeric HPV16L1-HPV16L2 VLP in both rabbits and mice also exhibited cross-neutralization to high-risk and low-risk HPV types evolutionarily divergent from HPV-16 [93]. One important and interesting issue in the development of chimeric VLP vaccines is to determine in future studies whether or not the slow decline of antibody titers observed with L1 VLP vaccines will also be exhibited by chimeric L1-L2 VLP vaccines. The inclusion of $\mathrm{L} 2$ as a vaccinogen represents a promising direction for next-generation preventive HPV vaccines in inducing broad-spectrum neutralizing antibodies against HPV.

\subsection{Therapeutic HPV vaccines}

Vaccination to control and prohibit the progression of HPV-associated lesions has been evaluated in a variety of pre-clinical models and clinical trials using live vector, peptide, protein, DNA, RNA replicon, and dendritic cell (DC)-based therapeutic HPV vaccines targeting HPV E6 and E7 oncoproteins (for review, see [94,95]).

5.2.1 Live vector-based therapeutic HPV vaccines-The concept of live vector vaccines encompasses the use of bacterial and viral vectors which replicate within the body and facilitate the spread of antigen. Live vector-based therapeutic HPV vaccines can deliver E6 and E7 antigens to antigen-presenting cells to stimulate antigen presentation through MHC class I to $\mathrm{CD}^{+}$cytotoxic $\mathrm{T}$ cells and $\mathrm{MHC}$ class II to $\mathrm{CD} 4^{+} \mathrm{T}$ helper cells. However, live vectors inherently pose a potential safety risk, particularly to immunocompromised individuals. Live vectors may also face limited capacity for repeated administration due to 
the induction of vector-specific neutralizing antibodies and/ or the possibility of pre-existing vector-specific immunity.

5.2.1.1 Bacterial vector-based vaccines: Several bacterial vectors have been explored for therapeutic HPV vaccines including Listeria monocytogenes [96,97], Lactobacillus casei [98], and Lactococcus lactis [99]. Among the many bacterial vectors, L. monocytogenes has generated significant interest. L. monocytogenes is a gram-positive intracellular bacterium that invades macrophages and evades phagocytosis within the phagosome by using listeriolysin O (LLO), a pore-forming toxin. Upon its escape into the cytoplasm of the APC, antigens are delivered and processed in both MHC class I and class II pathways that in turn activate $\mathrm{CD}^{+}$and $\mathrm{CD} 8^{+} \mathrm{T}$ cells. $L$. monocytogenes-based HPV E7 vaccines have been shown to resolve solid implanted tumors in transgenic mice with tissue-specific expression of HPV-16 E6 and E7 antigens by inducing a low- avidity CD8 ${ }^{+} \mathrm{T}$ cell response, thus overcoming central tolerance [96]. This finding suggests that the L. monocytogenes vector may be important in eliciting immune responses to tumors that attempt to escape a robust immune response.

These pre-clinical studies have translated to clinical trials. ADXS11-001, formerly known as Lovaxin C or Lm-LLO-E7, is a live, attenuated L. monocytogenes bacterial vector secreting HPV-16 E7 fused to LLO. In Phase I trials, ADXS11-001 was found to be safe and well tolerated with a dose-limiting toxicity linked to hypotension and flu-like symptoms $[100,101]$. Antigen-specific T-cell responses and some clinical responses were observed, which will be further assessed in Phase II clinical trials. ADXS11-001 is currently being studied in three clinical trials with active enrollment. An open-label, Phase I dose-escalation trial will assess the safety of ADXS11-001 in patients with HPV-associated oropharyngeal cancer (NCT01598792) [102]. Another ongoing Phase II trial will assess the safety and efficacy of ADXS11-001 in treating and increasing the one-year survival rate of women with persistent or recurrent cervical carcinoma (NCT01266460) [103]. A randomized, single-blind, placebo-controlled Phase II study is assessing whether three doses of ADXS11-001 in women with CIN2/3, for whom surgery is indicated, can safely reverse disease (NCT01116245) [104]. Recruitment of the first group of patients has recently been completed for this Phase II trial of ADXS11-001 in CIN 2/3 patients [105].

5.2.1.2 Viral vector-based: The high immunogenicity of viral vectors makes them attractive for use in therapeutic HPV vaccines. There have been several viral vectors used to deliver HPV E6 and E7 antigens, including adenoviruses [106,107], alphaviruses [108-110] and vaccinia virus [111-113]. The vaccinia virus, an enveloped, double-stranded DNA virus within the Poxviridae family, has emerged as a particularly promising viral vector of interest because of its large genome and high infectivity. Vaccinia-based vaccines have been explored in pre-clinical models utilizing intracellular targeting strategies for increased antigen presentation in DCs. These vaccines include vaccinia encoding E7 fused to calreticulin [113], vaccinia-expressing E7 fused to listeriolysin O [114], and vacciniaexpressing E7 linked to sorting signal and Lysosomal-associated membrane protein (SigE7LAMP-1) [114]. Additionally, vaccinia-based vaccines encoding SigE7LAMP and expressing human Fms-like tyrosine kinase 3 (Flt3) ligand was shown to have protective and therapeutic anti-tumor effects against E7-expressing tumors in vaccinated mice due to increased antigen-specific CD8 ${ }^{+} \mathrm{T}$ cell response detected by ELISPOT for IFN- $\gamma$ and E7 tetramer-staining assays [112].

Vaccinia vector-based therapeutic HPV vaccines have translated to clinical trials. A recombinant vaccinia virus expressing HPV-16 and HPV-18 E6/E7 antigens (TA-HPV) has been evaluated in Phase I/II clinical trials in patients with early-stage cervical cancer [115], late-stage cervical cancer, [116], vulvar intraepithelial neoplasia, [117], and vaginal 
intraepithelial neoplasia [118]. TA-HPV was found to be safe, well tolerated, and potent in stimulating vaccinia-specific antibody and HPV antigen-specific CTL responses $[115,116,119]$. A Phase II trial in patients with early-stage cervical cancer has been conducted to study the safety and immunological effects of TA-HPV in combination with surgery $[115,120]$. The results of this trial indicated that TA-HPV is safe, causing only mild to moderate toxicities, and was able to generate HPV-specific CTLs and serological responses in some patients [115].

Another vaccinia-based therapeutic HPV vaccine in clinical trials is MVA-E2, a recombinant modified vaccinia Ankara vector encoding bovine papillomavirus E2. E2 is the transcriptional repressor of E6 and E7 oncoproteins. For CIN lesions that have no viral integration into the host genome and therefore express HPV E2, MVA-E2 can bind to the HPV genome and prevent the upregulation of E6 and E7 oncoproteins, thus potentially controlling HPV-associated CIN lesions. Intrauterine administration of MVA-E2 vaccine was found to be well tolerated and increased antibody levels against both MVA virus and E2 protein in both patients with CIN [121,122] and genital warts [123]. However, there is no evidence that E2 expression contributes to the therapeutic effect as the vaccine may potentially function by establishing a proinflammatory environment in the cervicovaginal tract that induces an immune response.

The modified vaccinia Ankara vector which expresses HPV-16 E6 and E7 antigens and adjuvant IL-2 (MVA-HPV-IL2, also known as TG4001/R3484) has also been explored in clinical trials. TG4001/R3484 was designed with two goals in mind: i) alert the immune system specifically to HPV-16-infected cells that have started to undergo pre-cancerous transformation (cells presenting HPV-16 E6 and E7 antigens) and ii) further stimulate the immune system in clearing the infection through an adjuvant (IL-2). In Phase II clinical trials, TG4001/R3484 was found to be both safe and effective in producing clinical responses in women with HPV-16-positive CIN 2/3. Ten of 21 women in the trial no longer had detectable levels of CIN 2/3 six months after vaccination. At the twelve-month followup, no relapse or HPV-16 persistence was observed in these women [62]. TG4001/R3484 is currently in a placebo-controlled Phase IIb trial on patients with HPV-related CIN 2/3 lesions. Interim results demonstrated proof-of-concept for the therapeutic vaccine in HPV-16 monotherapy, but the trial did not reach its primary endpoint of six-month resolution in the CIN 2/3 indication and will not move on to a Phase III trial [124].

Vaccinia vector-based vaccines have also been assessed in clinical trials using heterologous prime boost regimens. In a Phase I clinical trial, 29 female patients with HPV-associated high-grade anogenital intraepithelial neoplasia received three doses of TA-CIN, a fusion protein-based HPV vaccine, followed by one dose of TA-HPV [125]. The vaccine was safe and well tolerated in patients without any significant adverse side effects. Moreover, full and partial clinical responses were seen in $17 \%$ of patients whereas $62 \%$ had symptomatic improvement. A separate Phase II trial of DNA vaccine with TA-HPV prime boost in combination with topical imiquimod in CIN3 patients is recruiting patients (NCT00788164) [126].

5.2.2 Peptide-based Therapeutic HPV vaccines—Direct administration of peptides derived from HPV antigens can lead to the uptake of peptides by dendritic cells for antigen processing and presentation, thus activating antigen-specific T-cell immunity. Peptide-based vaccines are stable, easy to produce, and have a high safety profile. They involve the identification of CTL and CD4 T-helper epitopes that allow for the stimulation of potent $\mathrm{CD} 8^{+}$and $\mathrm{CD} 4^{+} \mathrm{T}$-cell immune responses respectively. Research has focused on addressing the main limitations of peptide-based vaccines, namely their low immunogenicity, and the obstacle of MHC restriction. 
Adjuvants can be used to enhance the immunogenicity of peptide-based vaccines. Some immunostimulatory molecules that have been explored include immunoglobulin $\mathrm{G}$ fragment [127], immunomodulatory element comprised of streptavidin fused to the extracellular domain of murine 4-1BBL [128], and DC stimulatory cytokine Bryostatin [129]. Toll-Like Receptor (TLR) agonists have also been explored as adjuvants for peptide-based HPV vaccines because of their capability to activate both innate and adaptive immunity. For instance, a prime boost regimen of HPV-16 E7 (a.a.43 - 77), which contains both, a CTL epitope and a $\mathrm{T}_{\mathrm{H}}$ epitope, adjuvanted with TLR9 agonist $\mathrm{CpG}$ oligodeoxynucleotide (CpG $\mathrm{ODN}$ ) was shown to enhance $\mathrm{E} 7$-specific $\mathrm{CD}^{+}$and $\mathrm{CD}^{+} \mathrm{T}$ cells, detected by FACS analysis, and produce potent therapeutic anti-tumor effects and survival analysis against HPV-16-expressing tumors in vaccinated mice [130]. A vaccine with CTL epitope fused to a T-helper epitope, PADRE, along with $\mathrm{CpG}$ adjuvant produced specific $\mathrm{CD} 8^{+} \mathrm{T}$ cell responses and potent anti-tumor effects in mice with tumors expressing HPV-16 E7 [131]. Other TLR agonists explored in such experiments include the TLR4 agonist carrageenan [132] and TLR3 agonist Poly (I:C) [133]. Recently, a therapeutic peptide vaccination strategy (TriVax) consisting of intravenous administration of immunodominant CD8+ T-cell epitope HPV-16 E7 (aa49-57), mixed Poly (I:C) and costimulatory anti-CD40 monoclonal antibody was assessed. TriVax was found to induce large and persistent T-cell responses and induced tumor clearance against established HPV-16 E7-expressing tumors in two HPVinduced murine cancer models [134]. These results suggest that adjuvants targeting dendritic cells may potentiate peptide-based vaccines.

The enhancement of the immunogenicity of peptide-based vaccines has been explored in clinical studies. Because HLA-A*0201 is the most common human MHC class I molecule carried by over $50 \%$ of the general population, peptide-based therapeutic HPV vaccine studies have focused on human CTL epitopes encoding HPV-16 E7 that is presented by HLA-A*0201. As stated above, these CTL epitope peptide vaccines are often weakly immunogenic. Hence, supplementation of these peptide-based vaccines with a strong adjuvant as well as a strategy to recruit non-specific $\mathrm{T}$ cell help may boost $\mathrm{CD} 8+\mathrm{T}$ cell immune responses to the CTL epitope and result in clearance of HPV. The combination of CTL epitope peptide with non-specific T-cell help has been explored in the form of a lipopeptide construct of HPV-16 E7 aa86-93 peptide linked to PADRE peptide in women with late-stage cervical cancer [135]. Further similar studies have focused on combining two E7-derived epitopes (aa11-20 and aa 86-93) with Montanide ISA 51 as adjuvant in combination with PADRE universal T-helper peptide. However, among these trials, there were only six late-stage cervical cancer patients who mounted a weak immune response against the E7 86 - 93 peptide sequence, without evidence of anti-tumor benefits [136,137]. A Phase I clinical trial found that a vaccine consisting of HPV-16 E7 peptide (aa 12-20) administered along with a construct of Incomplete Freund's adjuvant, HPV E7 lipopeptide (aa 86-93) and PADRE, was able to stimulate an immune response in a significant proportion of eighteen HLA-A2-positive patients with CIN/VIN II/III [138]. The vaccine was found to be well tolerated and led to complete regression of CIN lesions in 3 of 17 evaluable patients. However, as these epitopes are HLA-A2*0201-restricted, these peptide vaccines would only have clinical benefit for the population of patients who are positive for this MHC class I.

The limitation of MHC restriction associated with peptide-based vaccines results in the need to identify immunogenic epitopes corresponding to the polymorphic MHC molecules within the population. Long overlapping peptides circumvent $\mathrm{MHC}$ restriction by including a range of antigenic epitopes of HPV E6 and E7 proteins and have renewed interest in therapeutic HPV E6/E7 peptide-based vaccines. These larger peptides have led to recent clinical trials in end-stage cervical cancer patients. A vaccine comprised of 13 overlapping peptides representing HPV-16 E6 and E7 antigens mixed with Montanide ISA 51 adjuvant was safe, 
well tolerated, and able to elicit a broad T-cell response in end-stage cervical cancer patients [139]. A second clinical trial using a broad array of epitopes in early-stage cervical cancer patients generated increased HPV-16-specific $\mathrm{CD} 4^{+}$and $\mathrm{CD} 8^{+} \mathrm{T}$-cell responses relative to patients who were not vaccinated [140]. Phase II clinical trials of this vaccine demonstrated great efficacy in HPV-16-positive high-grade VIN patients. Half of the patients with histologically confirmed HPV-16-positive VIN3 displayed a complete regression of their lesion after 3 or 4 vaccinations with HPV-16 E6/E7 overlapping peptide vaccine [141]. An investigation as to why vaccination did not lead to complete regression in all patients showed that a high ratio of the number of HPV16-specific vaccine-primed effector T cells to the number of HPV16-specific $\mathrm{CD} 4{ }^{+} \mathrm{CD} 25^{+} \mathrm{Foxp} 3^{+} \mathrm{T}$ reg cells was predictive of clinical success [142]. Foxp $3^{+} \mathrm{T}$ cells have been associated previously with impaired immunity in malignancies. Recently, a placebo-controlled randomized Phase II study demonstrated that this HPV-16 E6/E7 synthetic overlapping long-peptide vaccine increased the numbers of circulating IFN-Y-producing HPV-16-specific T cells in patients with HPV-16+ HSIL [143]. Similar to the earlier trials, vaccination had few side effects, with minor swelling at the injection site and flu-like symptoms. However, no HPV clearance was observed at the time of lesion excision, nor could conclusions be drawn on vaccine-induced T-cell infiltration of the lesion due, in part, to problems with patient accrual. It is important to take into consideration that patients with HSIL are generally asymptomatic and can be treated by minor surgical procedures. Hence, future developments should consider the disparities of side effects between standard of care and new therapies to maximize the potential benefits of overlapping peptide-based vaccination. The focus remains on developing a well-tolerated formulation capable of generating strong immune responses in patients with pre-malignant HPV-associated disease.

5.2.3 Protein-based vaccines-Protein-based vaccines have emerged as promising forms of therapeutic HPV vaccines. They are safer compared to live-vector based vaccines and can circumvent MHC restriction by including all epitopes that bind to all haplotypes of MHC class I and class II molecules. However, protein-based vaccines have some disadvantages such as relatively poor immunogenicity. In addition, protein-based vaccines are processed through the endocytic pathway and are presented via the MHC class II pathway, generating predominantly antibody responses rather than cytotoxic T-lymphocyte (CTL) responses. Thus, protein-based vaccines require strategies that increase its immunogenicity and increase induction of $\mathrm{CD} 8^{+}$cytotoxic $\mathrm{T}$-cell immune responses to promote anti-tumor immunity.

Numerous strategies to increase potency of protein-based vaccines have been studied, including the use of a diverse array of adjuvants and fusion proteins. These adjuvants include liposome-polycation-DNA (LPD) adjuvant [144], saponin-based ISCOMATRIX [145], and Toll-like receptor agonists [146]. In addition, fusion proteins comprised of HPV E7 fused to immunostimulatory molecules have been shown to enhance E7-specific T cellmediated immune responses, such as HPV E7 fused to DC-targeting molecules Bordetella pertussis adenylate cyclase [147], Mycobacteria-derived heat- shock proteins [148,149], and truncated Pseudomonas aeruginosa exotoxin A [150]. This strategy has also been explored in the context of a fusion protein vaccine comprised of HPV-16 E7 fused to the cellpenetrating peptide Limulus polyphemus protein to improve cross-presentation of antigen in DCs [151]. Furthermore, investigators have found that HPV-16 E7 linked to a bacterial lipid moiety administered in E7-expressing tumor-bearing mice induced appreciable E7-specific CTL responses, measured by ELISPOT and flow cytometry, and anti-tumor effect, measured by tumor volume [152]. Recently, the employment of adjuvants has been explored in combination with an improved delivery method to enhance protein-based vaccine potency. Kang et al. found that intramuscular injection of protein-based vaccines with TLR9 agonist $\mathrm{CpG}$, followed by electroporation, can lead to 3 major advantages: i) increased 
delivery of the protein-based vaccine into muscle cells, ii) enhanced antigen-specific CD8+ $\mathrm{T}$ cell immune responses, and iii) heighten potent anti-tumor effects; all of which result in enhanced protein-based HPV vaccine potency [146].

In clinical trials, several protein-based therapeutic HPV candidates have been explored. Among these candidates, HspE7 has generated considerable interest. HspE7 is a chimeric protein composed of bacille Calmette-Guerin heat-shock protein (Hsp65) and HPV-16 E7. HspE7 was previously found to be well tolerated as a single-agent therapy in both Phase I and Phase II clinical trials, generating lesion regression in several HPV-associated diseases, including recurrent respiratory papillomatosis [153], anal high-grade squamous intraepithelial lesions [154], and CIN 2/3 [155-157]. These trials began prior to the finding that adjuvant Poly-ICLC, a Toll-like receptor 3 agonist consisting of polyinosinicpolycytidylic acid stabilized with polylysine and carboxymethylcellulose, significantly strengthening HspE7 vaccine immunogenicity. However, it is not clear whether HspE7 with Poly-ICLC adjuvant vaccine has been further tested in clinical trials (NCT00493545) [158].

5.2.4 DNA vaccines-Among the different forms of therapeutic HPV vaccines, DNA vaccines have emerged as an attractive approach due to their safety, stability, and simplicity. DNA vaccination involves directly injecting plasmid DNA-encoding antigen of interest into host cells. This promotes expression and presentation of the encoded antigen by transfected cells and stimulates cell-mediated and/or humoral immune responses against the encoded antigen. DNA vaccines are safe and do not pose the intrinsic safety risks that are associated with introducing a tumor cell, bacteria, or virus into a patient. Naked DNA is easy to manufacture and can sustain expression of target cells for longer periods than RNA vaccines. In addition, DNA vaccines do not elicit neutralizing antibodies in vivo as do live vector-based vaccines, therefore, increasing the capacity for repeated administration. However, DNA vaccines have shown poor immunogenicity and require strategies to increase vaccine potency. Many strategies to enhance DNA vaccine potency have focused on targeting DNA vaccines to DCs, the potent activators of antigen-specific immune responses. In general, these strategies of improving the potency of therapeutic HPV DNA vaccines through modification of DCs can be classified by: i) increasing the number of antigen-expressing/antigen-loaded DCs, ii) improving HPV antigen expression, processing, and presentation in DCs, and iii) enhancing DC and T-cell interaction (for review, see [95]).

Encouraging data from pre-clinical studies have led to several therapeutic HPV DNA vaccines in clinical trials. Strategies to increase the number of antigen-expressing/-antigenloaded DCs include enhanced delivery methods targeting DNA vaccine to DCs. These delivery methods include gene gun, microencapsulation, and electroporation. For example, there is an ongoing, open-label, randomized clinical trial utilizing a clinical grade gene gun device that delivers pnGVL4a-CRT/E7 (Detox), a therapeutic HPV DNA vaccine, to treat high-grade cervical intraepithelial neoplasia (CIN 2/3) lesions in HPV-16 positive patients (NCT00988559) [159]. This clinical trial is a head-to-head comparison of the immunogenicity of three routes of administration (intradermal administration via gene gun, intramuscular administration, and intralesional delivery) of pnGVL4a-CRT/E7.

Microencapsulation has also been evaluated in several clinical trials [160-162] (for review, see [163]). Of particular interest is ZYC101, a plasmid encoding an HPV-16 E7 HLA-A2 restricted peptide encapsulated in $1-2 \mu \mathrm{m}$ biopolymer microparticles composed of polylactide co-glycolide (PLG). In a Phase 1 clinical trial examining the potential treatment of anal dysplasia with ZYC101, ten of twelve patients generated E7-specific immune responses and 3 out of 12 showed histological improvements [160]. Another Phase 1 trial studied the effect of ZYC101 on patients with high-grade CIN. Five of 15 patients experienced complete histological regression and 11 showed significant HPV-specific T-cell 
responses with no serious adverse effects [161]. A more recent version of the plasmid, amolimogene bepiplasmid (ZYC101a), encoding HPV-16 and -18 E6 and E7 fragments was used in a Phase II clinical trial involving 127 subjects with high-grade CIN. The vaccine was well tolerated and promoted CIN 2/3 resolution in patients under 25 years of age compared to the placebo group (70\% versus $23 \%$ ) [162]. Amolimogene was recently explored in a Phase II/III double-blinded, randomized, placebo-controlled clinical trial examining its efficacy and safety in the treatment of patients with CIN 2/3. Subjects with histologicevaluation confirmed CIN 2/3 lesions of the cervix were recruited to participate. After a diagnostic cervical punch biopsy confirming high grade CIN, eligible subjects were enrolled and randomized to either drug or placebo groups and, during the six-month study period, were monitored by colposcopic, cytologic, and HPV testing. Persistence or resolution of disease was determined by use of a loop electrosurgical excision procedure (LEEP) performed at study exit [164]. Results indicate that 11 of 21 patients receiving amolimogene generated an enhanced T-cell response to HLA-A2 restricted HPV 16/18 peptides compared to baseline and 6 of 21 subjects experienced resolution of CIN 2/3 lesions [164].

Besides microencapsulation, electroporation following intramuscular injection has also been assessed as a potential vaccine delivery method [165]. One such example is VGX-3100, a DNA vaccine incorporating plasmids targeting HPV-16 and 18 E6 and E7 proteins. The candidate vaccine is delivered via intramuscular injection followed by electroporation (EP) using a CELLECTRA constant current device to deliver a small electrical charge. VGX-3100 has shown promise in animal studies where treatment led to an increased immune response resulting in decreased tumor size. In a Phase I clinical trial, subjects with a history of high-grade CIN were vaccinated with VGX-3100. VGX-3100 immunization was well tolerated and elicited a T-cell response in 14 out of 18 clinical subjects and all subjects reported antibody positivity to at least two antigens [166]. Individuals with histologically confirmed HPV-16 and -18 associated high-grade CIN are currently being recruited for a double-blinded, randomized, placebo-controlled Phase II clinical trial (NCT01304524) [167].

Several clinical trials have studied the effects of vaccines designed to improve MHC class I processing. A Phase I clinical trial showed that 8 of 15 patients with high-grade CIN had increased E7-specific T-cell responses after intramuscular injection with pNGVL4a-Sig/ E7(Detox)/HSP70 (a vaccine consisting of DNA encoding a signal sequence localized to the endoplasmic reticulum (Sig), linked to an attenuated form of HPV-16 E7 fused to HSP70). Although the vaccine was well tolerated with no dose-limiting toxicities, subjects treated with the vaccine did not experience significantly enhanced therapeutic effects as compared to unvaccinated subjects [168]. Despite poor immunogenicity, complete histologic regression did occur in $33 \%$ of patients vaccinated with the highest dose of pNGVLA4a-Sig/ E7(detox)/ HSP70 (3 mg). The same vaccine has also been used in patients with HPV-16+ head and neck cancer (Gillison and $\mathrm{Wu}$, personal communication). It is now clear that approximately $20 \%$ of head and neck cancers are associated with HPV, particularly HPV-16 (for review, see [169]). These early phase clinical trials with pNGVLA4a-Sig/E7(detox)/ HSP70 demonstrate great safety without significant side effects and have promising applications to both cervical cancers as well as head and neck cancers caused by HPV. The results of the early phase clinical trials suggest that prime-boost with different expression vectors or delivery methods such as electroporation or intradermal administration via gene gun may be necessary to increase DNA vaccine potency.

Though the aforementioned techniques have been effective, other less invasive methods have shown promise in improving immunogenicity. Of particular interest are TLR agonists, immunomodulators that generate robust immune responses and increased potency when used in conjunction with therapeutic HPV vaccines. Notably, imiquimod has been 
repeatedly examined in clinical trials. A TLR7 agonist, imiquimod promotes the activation of antigen-presenting cells leading to the production of cytokines IFN-alpha, IL-6, and TNFalpha [170]. Cytokine production induces a robust and potent immune response by facilitating adaptive immune cell activation and differentiation. Thus, imiquimod represents a potentially promising adjuvant for therapeutic HPV DNA vaccines. For example, imiquimod was used as an adjuvant for a HPV DNA vaccine that encoded a calreticulin (CRT) and HPV-16 (E7) fusion protein (CRT/E7) administered to C57BL/6 mice expressing tumors [171]. Experimental mice treated with CRT/E7 DNA and imiquimod were found to have an increased frequency of E7-specific CD8+ T cells within their spleens as compared to the frequency found within the spleens of control mice (CRT/E7 DNA vaccination only). Moreover, mice receiving combined therapy experienced tumor reduction and prolonged survival when compared to mice receiving CRT/ E7 DNA vaccination only. Furthermore, cells expressing NK1.1, namely NK and NKT cells, were found with increased frequency within the tumor microenvironment suggesting that they play a key role in tumor volume reduction. Of note, combined treatment of DNA encoding CRT/E7 and imiquimod also reduced the number of myeloid-derived suppressor cells that have an immunosuppressive role in the tumor microenvironment. Therefore, the use of imiquimod as an adjuvant for therapeutic DNA vaccines may have multiple mechanisms to improve anti-tumor effects.

The encouraging pre-clinical data has lead to an ongoing Phase I clinical trial which investigates a prime-boost strategy combined with topical imiquimod in treating women with CIN3. The subjects were intramuscularly primed with pNGVL4a-Sig/E7(detox)/ HSP70 and then boosted with a recombinant vaccinia virus encoding HPV-16 and 18 E6 and E7 (TA-HPV) with topological application of imiquimod on the CIN lesion [126]. In addition, imiquimod has been used in a Phase II clinical trial in conjunction with TA-CIN, a fusion protein-based vaccine expressing HPV-16 L2-E6-E7 conjugated proteins, in patients with high-grade vulvar intra-epithelial neoplasia (VIN) [33]. Intramuscular administration of TA-CIN and topical application of imiquimod was well tolerated without adverse effects. Antigen-specific antibody titers were generated; however, titers were not significantly different after imiquimod application or vaccination in "responders" and "non-responders." "Responders" to the therapy demonstrated high levels of $\mathrm{CD}^{+}$and $\mathrm{CD} 8^{+} \mathrm{T}$ cells locally as well as within HPV-associated lesions. Imiquimod was shown to increase T- cell infiltration, leading to the complete regression of VIN lesions in $63 \%$ of patients one year post treatment. In addition, $36 \%$ of the patients with VIN lesions showed complete HPV clearance and $79 \%$ of women remained symptom-free. Phase III clinical trials will be needed to assess the comparative efficacy of this combinatorial approach.

5.2.5 Dendritic cell-based vaccines-Dendritic cells are professional antigenpresenting cells and are thus able to induce the adaptive immune response, particularly $\mathrm{T}$ cell-mediated immune responses, by processing antigen and priming $\mathrm{T}$ cells in the context of both MHC class I and MHC class II pathways. The process of pulsing DCs with HPV antigenic peptides, proteins, or DNA-encoding antigens ex vivo, which enables loading of MHC class I and class II molecules with HPV epitopes, allows DCs to differentiate and mature. Upon re-administration of the DCs into the body, T cells become primed and elicit a cell-mediated immune response. Understanding DC differentiation, maturation, as well as antigen processing and presentation have proven fruitful in providing a rationale for improving DC-based vaccines.

A Phase I clinical trial of a DC-based HPV vaccine using HPV-16 E7 and/or HPV-18 E7 assessed the safety and immunogenicity of the therapeutic vaccine in 10 patients diagnosed with early-stage cervical cancer [172]. All the patients generated antibody and CD4 ${ }^{+}$T-cell responses to the HPV E7-loaded DC vaccination and 8 of the 10 patients developed increased $\mathrm{E} 7$-specific $\mathrm{CD} 8^{+} \mathrm{T}$ cell counts compared to pre-vaccination levels. Overall, the 
vaccine was determined to be safe and immunogenic. Although DC-based vaccines may be used in advanced cases of cervical cancer or in combination with other therapies, it is unlikely they will be used to treat CIN lesions because the procedures involved in this kind of treatment are quite labor-intensive and costly.

5.2.6 Combination strategies-Although strategies to enhance different types of therapeutic vaccines have been developed, it is likely that the combination of strategies will further increase immunogenicity and efficacy of therapeutic HPV vaccines. Hence, therapeutic HPV vaccine strategies have shifted in the direction of combinatorial approaches to work toward commercialization. One combinatorial approach uses fusion protein antigens in concurrence with low-dose radiation treatment of tumors in pre-clinical models [173]. Vaccination of mice with calreticulin (CRT) fused with HPV-16 E7 combined with radiation therapy showed an increase in therapeutic efficacy as compared to vaccinated mice alone. The combination of therapies elicited the highest frequency E7-specific $\mathrm{CD}^{+} \mathrm{T}$-cell response and increased tumor susceptibility to E7-specific CTL activity in the tumormicroenvironment. This response slowed and stabilized tumor growth, which ultimately led to an increase in mouse long-term survival rates. In addition, radiation therapy was successful in causing apoptosis of tumor cells, indicating that radiation is a useful method in stabilizing tumor-cell growth when applied with immunotherapy. The combination of chemotherapy, radiation therapy, and vaccination suggest effective anti-tumor effects.

Chemotherapeutic agents in combination with DNA-based vaccines is another emerging combination that may be an effective HPV therapy as shown in pre-clinical models [174178]. For example, the chemotherapeutic agent, apigenin, was used concurrently with a DNA-encoding heat-shock protein 70 (HSP70) and HPV-16 E7 [179]. Vaccination of E7HSP70 DNA in conjunction with apigenin chemotherapy demonstrated the highest frequency of effector $\mathrm{CD} 8^{+} \mathrm{T}$ cells and memory $\mathrm{CD} 8^{+} \mathrm{T}$ cells. Vaccination and chemotherapy caused tumor susceptibility to E7-specific cytotoxic immune responses that led to a reduction in tumor size and an increase in survival rates. Apigenin treatment also proved to increase tumor cell apoptosis in a dose-dependent manner. Overall, the combination of chemotherapy and DNA-based HPV vaccination generated the greatest antitumor effect.

Promising pre-clinical data has led to the concurrent evaluation of several therapeutic HPV vaccine candidates in early phase clinical trials as summarized in Table 2.

\section{Expert opinion}

Significant steps toward the ideal prophylactic and therapeutic vaccines have been made. The effectiveness of commercially available VLP vaccines in generating a short-term antibody response to HPV-16 and HPV-18 is evident. However, future generations of preventive vaccines must focus on lowering cost to make them more accessible to susceptible populations in developing countries. This will make the largest impact on the incidence of persistent HPV infection and cervical cancer. Ineffective cross-protection and cost-effectiveness are two additional problems that face prophylactic vaccines. Although it is feasible that ongoing clinical trials of the Merck V503 preventive nonavalent vaccine may effectively provide broader protection, cost may still be an issue inhibiting the use of the vaccine in countries that carry the majority of the burden of cervical cancer. Therefore, cost is the most pertinent issue facing future preventive vaccines.

Antigen-specific immunotherapy generated by therapeutic HPV vaccines has promise for the treatment of cervical cancer and clearance of HPV infection. However, progression of therapeutic HPV vaccines through clinical trials has not been as robust compared to the 
commercialization of preventive HPV vaccines. In addition, it would seem that women with a lower disease burden and pre-invasive disease are more logical candidates for an antigenspecific immunotherapy than women with bulky invasive disease, prior chemotherapy, poor performance status, and profound immunosuppression. Therefore, the progress of therapeutic HPV vaccines through clinical trials may likely require a combination strategy of different therapeutic modalities. In addition, therapeutic efficacy might improve by addressing the immunosuppressive tumor microenvironment to improve the association of immune response and clinical outcomes. Strategies could be used in conjunction with existing live vector, protein/peptide, DC-based vaccines and DNA vaccine strategies to control tumor metastasis and eliminate cancer cells altogether. As research in the development of prophylactic and therapeutic HPV vaccines continues, different strategies will be created that will push human papillomavirus-associated malignancies closer to eradication.

\section{Acknowledgments}

This review is not intended to be an encyclopedic one, and the authors apologize to those works not cited.

\section{Bibliography}

Papers of special note have been highlighted as either of interest $(\bullet)$ or of considerable interest $(\bullet)$ to readers.

1. Hoory T, Monie A, Gravitt P, et al. Molecular epidemiology of human papillomavirus. J Formos Med Assoc. 2008; 107(3):198-217. [PubMed: 18400605]

2. de Martel C, Ferlay J, Franceschi S, et al. Global burden of cancers attributable to infections in 2008: a review and synthetic analysis. Lancet Oncol. 2012; 13(6):607-15. [PubMed: 22575588]

3. Parkin DM, Bray F. Chapter 2: the burden of HPV-related cancers. Vaccine. 2006; 3124(Suppl 3):S3/11-25. [PubMed: 16949997]

4. Bernard HU, Burk RD, Chen Z, et al. Classification of papillomaviruses (PVs) based on $189 \mathrm{PV}$ types and proposal of taxonomic amendments. Virology. 2010; 25401(1):70-9. [PubMed: 20206957]

5•. Munoz N, Bosch FX, de Sanjose S, et al. Epidemiologic classification of human papillomavirus types associated with cervical cancer. N Engl J Med. 2003; 6348(6):518-27. A landmark study on the importance of differential carcinogenicity of HPV types on the epidemiologic classification of HPV. [PubMed: 12571259]

6. Cogliano V, Baan R, Straif K, et al. Carcinogenicity of human papillomaviruses. Lancet Oncol. 2005; 6(4):204. [PubMed: 15830458]

7. Schiffman M, Clifford G, Buonaguro FM. Classification of weakly carcinogenic human papillomavirus types: addressing the limits of epidemiology at the borderline. Infect Agent Cancer. 2009; 4:8. [PubMed: 19486508]

8. zur Hausen H. Papillomaviruses and cancer: from basic studies to clinical application. Nat Rev Cancer. 2002; 2(5):342-50. [PubMed: 12044010]

9. Guan P, Howell-Jones R, Li N, et al. Human papillomavirus types in 115,789 HPV-positive women: a meta-analysis from cervical infection to cancer. Int J Cancer. 2012; 131(10):2349-59. [PubMed: 22323075]

10. Chaturvedi AK. Beyond cervical cancer: burden of other HPV-related cancers among men and women. J Adolesc Health. 2010; 46(4 Suppl):S20-6. [PubMed: 20307840]

11. Doorbar J. Molecular biology of human papillomavirus infection and cervical cancer. Clin Sci (Lond). 2006; 110(5):525-41. [PubMed: 16597322]

12•. Kines RC, Thompson CD, Lowy DR, et al. The initial steps leading to papillomavirus infection occur on the basement membrane prior to cell surface binding. Proc Natl Acad Sci USA. 2009; 106(48):20458-63. An important study elucidating the mechanism by which HPV infects the anogenital tract. [PubMed: 19920181] 
13. Einstein MH, Schiller JT, Viscidi RP, et al. Clinician's guide to human papillomavirus immunology: knowns and unknowns. Lancet Infect Dis. 2009; 9(6):347-56. [PubMed: 19467474]

14. Lehoux M, D’Abramo CM, Archambault J. Molecular mechanisms of human papillomavirusinduced carcinogenesis. Public Health genomics. 2009; 12(5-6):268-80. [PubMed: 19684440]

15. Klingelhutz AJ, Roman A. Cellular transformation by human papillomaviruses: lessons learned by comparing high- and low-risk viruses. Virology. 2012; 424(2):77-98. [PubMed: 22284986]

16. Parkin DM, Bray F, Ferlay J, et al. Global cancer statistics, 2002. CA Cancer J Clin. 2005; 55(2): 74-108. [PubMed: 15761078]

17. Jemal A, Bray F, Center MM, et al. Global cancer statistics. CA Cancer J Clin. 2011 Mar-Apr; 61(2):69-90. [PubMed: 21296855]

18. Guan P, Howell-Jones R, Li N, et al. Human papillomavirus types in 115,789 HPV-positive women: a meta-analysis from cervical infection to cancer. Int J Cancer. 2012; 131(10):2349-59. [PubMed: 22323075]

19. Schiller JT, Castellsague X, Villa LL, et al. An update of prophylactic human papillomavirus L1 virus-like particle vaccine clinical trial results. Vaccine. 2008; 26(Suppl 10):K53-61. [PubMed: 18847557]

20. Wright TC Jr, Cox JT, Massad LS, et al. 2001 Consensus guidelines for the management of women with cervical cytological abnormalities. JAMA. 2002; 287(16):2120-9. [PubMed: 11966387]

21. Bharti AC, Shukla S, Mahata S, et al. Anti-human papillomavirus therapeutics: facts \& future. Indian J Med Res. 2009; 130(3):296-310. [PubMed: 19901439]

22. Rose PG, Blessing JA, Gershenson DM, et al. Paclitaxel and cisplatin as first-line therapy in recurrent or advanced squamous cell carcinoma of the cervix: a gynecologic oncology group study. J Clin Oncol. 1999; 17(9):2676-80. [PubMed: 10561341]

23. Morris M, Eifel PJ, Lu J, et al. Pelvic radiation with concurrent chemotherapy compared with pelvic and para-aortic radiation for high-risk cervical cancer. N Engl J Med. 1999; 340(15):113743. [PubMed: 10202164]

24. Kyrgiou M, Koliopoulos G, Martin-Hirsch P, et al. Obstetric outcomes after conservative treatment for intraepithelial or early invasive cervical lesions: systematic review and meta-analysis. Lancet. 2006; 367(9509):489-98. [PubMed: 16473126]

25. Venturoli S, Ambretti S, Cricca M, et al. Correlation of high-risk human papillomavirus genotypes persistence and risk of residual or recurrent cervical disease after surgical treatment. J Med Virol. 2008; 80(8):1434-40. [PubMed: 18551620]

26. Coremans G, Snoeck R. Cidofovir: clinical experience and future perspectives on an acyclic nucleoside phosphonate analog of cytosine in the treatment of refractory and premalignant HPVassociated anal lesions. Expert Opin Pharmacother. 2009; 10(8):1343-52. [PubMed: 19463071]

27. Scheinfeld N, Lehman DS. An evidence-based review of medical and surgical treatments of genital warts. Dermatol Online J. 2006; 12(3):5. [PubMed: 16638419]

28. Beglin M, Melar-New M, Laimins L. Human papillomaviruses and the interferon response. J interferon Cytokine Res. 2009; 29(9):629-35. [PubMed: 19715460]

29. Yang J, Pu YG, Zeng ZM, et al. Interferon for the treatment of genital warts: a systematic review. BMC Infect Dis. 2009; 9:156. [PubMed: 19772554]

30. Cirelli R, Tyring SK. Interferons in human papillomavirus infections. Antiviral Res. 1994; $24(2-$ 3):191-204. [PubMed: 7526791]

31. Miller RL, Gerster JF, Owens ML, et al. Imiquimod applied topically: a novel immune response modifier and new class of drug. Int J Immunopharmacol. 1999; 21(1):1-14. [PubMed: 10411278]

32. Suzuki H, Wang B, Shivji GM, et al. Imiquimod, a topical immune response modifier, induces migration of Langerhans cells. J Invest Dermatol. 2000; 114(1):135-41. [PubMed: 10620129]

33. Daayana S, Elkord E, Winters U, et al. Phase II trial of imiquimod and HPV therapeutic vaccination in patients with vulval intraepithelial neoplasia. Br J Cancer. 2010; 102(7):1129-36. [PubMed: 20234368]

34••. Koutsky LA, Ault KA, Wheeler CM, et al. A controlled trial of a human papillomavirus type 16 vaccine. N Engl J Med. 2002; 347(21):1645-51. The first efficacy test of an HPV vaccine in humans, demonstrating its safety and proving in principle its ability to reduce the incidence of persistant HPV infection and cervical cancer. [PubMed: 12444178] 
35••. Harper DM, Franco EL, Wheeler C, et al. Efficacy of a bivalent L1 virus-like particle vaccine in prevention of infection with human papillomavirus types 16 and 18 in young women: a randomised controlled trial. Lancet. 2004 Nov 13-19; 364(9447):1757-65. Clinical evidence of the safety and efficacy of the AS04 adjuvant used in conjunction with the bivalent HPV 16/ 18 vaccine, serving as the foundation for Cervarix. [PubMed: 15541448]

36. Harper DM. Currently approved prophylactic HPV vaccines. Expert Rev Vaccines. 2009; 8(12): 1663-79. [PubMed: 19943762]

37. Lin K, Doolan K, Hung CF, et al. Perspectives for preventive and therapeutic HPV vaccines. J Formos Med Assoc. 2010; 109(1):4-24. [PubMed: 20123582]

38. D'Souza G, Dempsey A. The role of HPV in head and neck cancer and review of the HPV vaccine. Prev Med. 2011; 53(Suppl 1):S5-S11. [PubMed: 21962471]

39. Lowy DR, Schiller JT. Reducing HPV-associated cancer globally. Cancer Prev Res (Phila). 2012; 5(1):18-23. [PubMed: 22219162]

40. Harper DM, Williams KB. Prophylactic HPV vaccines: current knowledge of impact on gynecologic premalignancies. Discov Med. 2010; 10(50):7-17. [PubMed: 20670593]

41. Campo MS, Roden RB. Papillomavirus prophylactic vaccines: established successes, new approaches. J Virol. 2010; 84(3):1214-20. [PubMed: 19906917]

42. Schwarz TF. Clinical update of the AS04-adjuvanted human papillomavirus-16/18 cervical cancer vaccine, Cervarix. Adv Ther. 2009; 26(11):983-98. [PubMed: 20024678]

43. Giannini SL, Hanon E, Moris P, et al. Enhanced humoral and memory B cellular immunity using HPV16/ 18 L1 VLP vaccine formulated with the MPL/aluminium salt combination (AS04) compared to aluminium salt only. Vaccine. 2006; 24(33-34):5937-49. [PubMed: 16828940]

44. Ault KA. Quadrivalent vaccine against human papillomavirus to prevent high-grade cervical lesions. N Engl J Med. 2007; 356(19):1915-27. [PubMed: 17494925]

45. Ault KA. Effect of prophylactic human papillomavirus L1 virus-like-particle vaccine on risk of cervical intraepithelial neoplasia grade 2 , grade 3 , and adenocarcinoma in situ: a combined analysis of four randomised clinical trials. Lancet. 2007; 369(9576):1861-8. [PubMed: 17544766]

46. Paavonen J, Jenkins D, Bosch FX, et al. Efficacy of a prophylactic adjuvanted bivalent L1 viruslike-particle vaccine against infection with human papillomavirus types 16 and 18 in young women: an interim analysis of a phase III double-blind, randomised controlled trial. Lancet. 2007; 369(9580):2161-70. [PubMed: 17602732]

47. Paavonen J, Naud P, Salmeron J, et al. Efficacy of human papillomavirus (HPV)-16/18 AS04adjuvanted vaccine against cervical infection and precancer caused by oncogenic HPV types (PATRICIA): final analysis of a double-blind, randomised study in young women. Lancet. 2009; 374(9686):301-14. A clinical trial demonstrating the high efficacy of the HPV16/ 18 AS04adjuvanted vaccine against CIN2+ lesions associated with HPV 16/18 and non-vaccine oncogenic HPV types including HPV 31, 33 and 45. [PubMed: 19586656]

48. Dillner J, Kjaer SK, Wheeler CM, et al. Four year efficacy of prophylactic human papillomavirus quadrivalent vaccine against low grade cervical, vulvar, and vaginal intraepithelial neoplasia and anogenital warts: randomised controlled trial. BMJ. 2010; 341:c3493. [PubMed: 20647284]

49. Goldstone SE, Vuocolo S. A prophylactic quadrivalent vaccine for the prevention of infection and disease related to HPV-6, -11, -16 and -18. Expert Rev Vaccines. 2012; 11(4):395-406. [PubMed: 22551023]

50. Joura EA, Leodolter S, Hernandez-Avila M, et al. Efficacy of a quadrivalent prophylactic human papillomavirus (types 6, 11, 16, and 18) L1 virus-like-particle vaccine against high-grade vulval and vaginal lesions: a combined analysis of three randomised clinical trials. Lancet. 2007; 369(9574):1693-702. [PubMed: 17512854]

51. Joura EA, Garland SM, Paavonen J, et al. Effect of the human papillomavirus (HPV) quadrivalent vaccine in a subgroup of women with cervical and vulvar disease: retrospective pooled analysis of trial data. BMJ. 2012; 344:e1401. [PubMed: 22454089]

52. Garland SM, Hernandez-Avila M, Wheeler CM, et al. Quadrivalent vaccine against human papillomavirus to prevent anogenital diseases. N Engl J Med. 2007; 356(19):1928-43. An important Phase III clinical trial demonstrating the efficacy of the quadrivalent vaccine for preventing anogenital diseases on a large scale. [PubMed: 17494926] 
53•. Palefsky JM, Giuliano AR, Goldstone S, et al. HPV vaccine against anal HPV infection and anal intraepithelial neoplasia. N Engl J Med. 2011; 365(17):1576-85. A clinical trial demonstrating the safety and efficacy of the quadrivalent against anal intraepithelial neoplasia in men who have sex with men. [PubMed: 22029979]

54•. Giuliano AR, Palefsky JM, Goldstone S, et al. Efficacy of quadrivalent HPV vaccine against HPV Infection and disease in males. N Engl J Med. 2011; 364(5):401-11. A clinical trial proving that the quadrivalent vaccine prevents infection with HPV 6, 11, 16 and 18 and prevents related external genital lesions in males. [PubMed: 21288094]

55. Ma B, Roden R, Wu TC. Current status of human papillomavirus vaccines. J Formos Med Assoc. 2010; 109(7):481-3. [PubMed: 20677402]

56. Bharadwaj M, Hussain S, Nasare V, et al. HPV \& HPV vaccination: issues in developing countries. Indian J Med Res. 2009; 130(3):327-33. [PubMed: 19901442]

57. Garnett GP, Kim JJ, French K, et al. Chapter 21: modelling the impact of HPV vaccines on cervical cancer and screening programmes. Vaccine. 2006; 24(Suppl 3):S3/178-86.

58. Romanowski B, Schwarz TF, Ferguson LM, et al. Immunogenicity and safety of the HPV-16/ 18 AS04-adjuvanted vaccine administered as a 2-dose schedule compared with the licensed 3-dose schedule: results from a randomized study. Hum Vaccin. 2011; 7(12):1374-86. [PubMed: 22048171]

59. Dorans K. Fewer shots proposed to increase uptake of HPV vaccine. Nat Med. 2010; 16(8):832-3. [PubMed: 20689528]

60. Rowhani-Rahbar A, Carter JJ, Hawes SE, et al. Antibody responses in oral fluid after administration of prophylactic human papillomavirus vaccines. J Infect Dis. 2009; 200(9):1452-5. [PubMed: 19698077]

61. Frazer IH. Measuring serum antibody to human papillomavirus following infection or vaccination. Gynecol Oncol. 2010; 118(1 Suppl):S8-11. [PubMed: 20494221]

62. Brun JL, Dalstein V, Leveque J, et al. Regression of high-grade cervical intraepithelial neoplasia with TG4001 targeted immunotherapy. Am J Obstet Gynecol. 2011; 204(2):169, e1-8. [PubMed: 21284968]

63•. Roteli-Martins C, Naud P, De Borba P, et al. Sustained immunogenicity and efficacy of the HPV-16/ 18 AS04-adjuvanted vaccine: up to 8. 4 years of follow-up. Hum Vaccin Immunother. 2012; 8(3):390-7. A study indicating the duration of immunogencitiy generated byt the HPV-16/18 AS04-adjuvanted vaccine. [PubMed: 22327492]

64. Einstein MH, Baron M, Levin MJ, et al. Comparison of the immunogenicity and safety of Cervarix and Gardasil human papillomavirus (HPV) cervical cancer vaccines in healthy women aged 18-45 years. Hum Vaccin. 2009; 5(10):705-19. [PubMed: 19684472]

65. Einstein MH, Baron M, Levin MJ, et al. Comparative immunogenicity and safety of human papillomavirus (HPV)-16/ 18 vaccine and HPV-6/11/16/18 vaccine: follow-up from months 12-24 in a phase III randomized study of healthy women aged 18-45 years. Hum Vaccin. 2011; 7(12): 1343-58. [PubMed: 22048173]

66. Wheeler CM, Castellsague X, Garland SM, et al. Cross-protective efficacy of HPV-16/18 AS04adjuvanted vaccine against cervical infection and precancer caused by non-vaccine oncogenic HPV types: 4-year end-of-study analysis of the randomised, double-blind PATRICIA trial. Lancet Oncol. 2012; 13(1):100-10. [PubMed: 22075170]

67. Lehtinen M, Paavonen J, Wheeler CM, et al. Overall efficacy of HPV-16/ 18 AS04-adjuvanted vaccine against grade 3 or greater cervical intraepithelial neoplasia: 4-year end-of-study analysis of the randomised, double-blind PATRICIA trial. Lancet Oncol. 2012; 13(1):89-99. [PubMed: 22075171]

68. Stanley M. Immunobiology of HPV and HPV vaccines. Gynecol Oncol. 2008; 109(2 Suppl):S1521. [PubMed: 18474288]

69. Merck. ClinicalTrials.gov [Internet]. National Library of Medicine (US); Bethesda (MD): 2009. A phase iii clinical trial to study the immunogenicity, tolerability, and manufacturing consistency of V503 (A Multivalent Human Papillomavirus [HPV] L1 Virus-Like Particle [VLP] Vaccine) in preadolescents and adolescents (9 to 15 Year Olds) with a comparison to young women (16 to 26 Year Olds). 
70. Merck. ClinicalTrials.gov [Internet]. National Library of Medicine (US); Bethesda (MD): Oct 29. 2007 A randomized, double-blinded, tolerability and immunogenicity study of a multivalent human papillomavirus (HPV) L1 Virus-Like Particle (VLP) vaccine administered concomitantly with GARDASIL to 16- to 26- Year-Old women.

71. Merck. ClinicalTrials.gov [Internet]. National Library of Medicine (US); Bethesda (MD): Aug 22. 2007 A phase IIa randomized, double-blind controlled with gardasil, clinical trial to study thetolerability and immunogenicity of V505 (a Multivalent Human Papilloma Virus [HPV] L1 Virus Like Particle [VLP] Vaccine) in healthy 16 to 26 year old women.

72. Merck. ClinicalTrials.gov [Internet]. National Library of Medicine (US); Bethesda (MD): Oct 12. 2007 A randomized, international, double-blinded (With In-House Blinding), controlled with GARDASIL, dose-ranging, tolerability, immunogenicity, and efficacy study of a multivalent human papillomavirus (HPV) L1 Virus-Like Particle (VLP) vaccine administered to 16- to 26year-old women.

73. Li M, Cripe TP, Estes PA, et al. Expression of the human papillomavirus type $11 \mathrm{~L} 1$ capsid protein in Escherichia coli: characterization of protein domains involved in DNA binding and capsid assembly. J Virol. 1997; 71(4):2988-95. [PubMed: 9060658]

74. Rose RC, White WI, Li M, et al. Human papillomavirus type 11 recombinant L1 capsomeres induce virus-neutralizing antibodies. J Virol. 1998; 72(7):6151-4. [PubMed: 9621080]

75. Fraillery D, Baud D, Pang SY, et al. Salmonella enterica serovar Typhi Ty21a expressing human papillomavirus type $16 \mathrm{~L} 1$ as a potential live vaccine against cervical cancer and typhoid fever. Clin Vaccine Immunol. 2007; 14(10):1285-95. [PubMed: 17687110]

76. Schadlich L, Senger T, Gerlach B, et al. Analysis of modified human papillomavirus type 16 L1 capsomeres: the ability to assemble into larger particles correlates with higher immunogenicity. J Virol. 2009; 83(15):7690-705. [PubMed: 19457985]

77. Karanam B, Jagu S, Huh WK, et al. Developing vaccines against minor capsid antigen L2 to prevent papillomavirus infection. Immunol Cell Biol. 2009 May-Jun;87(4):287-99. [PubMed: 19421199]

78. Embers ME, Budgeon LR, Pickel M, et al. Protective immunity to rabbit oral and cutaneous papillomaviruses by immunization with short peptides of L2, the minor capsid protein. J Virol. 2002; 76(19):9798-805. [PubMed: 12208958]

79. Gaukroger JM, Chandrachud LM, O'Neil BW, et al. Vaccination of cattle with bovine papillomavirus type 4 L2 elicits the production of virus-neutralizing antibodies. J Gen Virol. 1996; 77(Pt 7):1577-83. [PubMed: 8758002]

80•. Roden RB, Yutzy WHt, Fallon R, et al. Minor capsid protein of human genital papillomaviruses contains subdominant, cross-neutralizing epitopes. Virology. 2000; 270(2):254-7. A landmark study showing that the minor capsid protein, L2, of HPV contains cross-reactive epitopes, indicating the potential for an L2-based vaccine to provide broad-spectrum protection. [PubMed: 10792983]

81. Pastrana DV, Gambhira R, Buck CB, et al. Cross-neutralization of cutaneous and mucosal Papillomavirus types with anti-sera to the amino terminus of L2. Virology. 2005; 337(2):365-72. [PubMed: 15885736]

82•. Kawana K, Yoshikawa H, Taketani Y, et al. Common neutralization epitope in minor capsid protein L2 of human papillomavirus types 16 and 6. J Virol. 1999; 73(7):6188-90. An important study indicating a common, cross-neutralizing L2 epitope between HPV 6 and 16. [PubMed: 10364381]

83. Gambhira R, Karanam B, Jagu S, et al. A protective and broadly cross-neutralizing epitope of human papillomavirus L2. J Virol. 2007; 81(24):13927-31. A study documenting the generation of a monoclonal antibody that binds to highly conserved L2 residues and its ability to neutralize pseudoviruses HPV 5, 6, 16, 18, 31, 45, 52, 58, bovine papillomavirus 1, and HPV11 native virions. [PubMed: 17928339]

84. Gambhira R, Jagu S, Karanam B, et al. Protection of rabbits against challenge with rabbit papillomaviruses by immunization with the $\mathrm{N}$ terminus of human papillomavirus type 16 minor capsid antigen L2. J Virol. 2007; 81(21):11585-92. [PubMed: 17715230] 
85. Yoon SW, Lee TY, Kim SJ, et al. Oral administration of HPV-16 L2 displayed on Lactobacillus casei induces systematic and mucosal cross-neutralizing effects in Balb/c mice. Vaccine. 2012; 30(22):3286-94. [PubMed: 22426329]

86. Tumban E, Peabody J, Peabody DS, et al. A pan-HPV vaccine based on bacteriophage PP7 VLPs displaying broadly cross-neutralizing epitopes from the HPV minor capsid protein, L2. PloS One. 2011; 6(8):e23310. [PubMed: 21858066]

87• do Caldeira JC, Medford A, Kines RC, et al. Immunogenic display of diverse peptides, including a broadly cross-type neutralizing human papillomavirus L2 epitope, on virus-like particles of the RNA bacteriophage PP7. Vaccine. 2010; 28(27):4384-93. An important study of the therapeutic platform utilizing PP7 bacteriophage to display immunogenic peptides. [PubMed: 20434554]

88••. Jagu S, Karanam B, Gambhira R, et al. Concatenated multitype L2 fusion proteins as candidate prophylactic pan-human papillomavirus vaccines. J Natl Cancer Inst. 2009; 101(11):782-92. A landmark study demonstrating the potency and potential of concatenated L2 proteins as panoncogenic HPV vaccines, forming the foundation for a clinical trial in India. [PubMed: 19470949]

89. Jagu S, Kwak K, Garcea RL, et al. Vaccination with multimeric L2 fusion protein and L1 VLP or capsomeres to broaden protection against HPV infection. Vaccine. 2010; 28(28):4478-86. [PubMed: 20434552]

90. Tuma RS. Assessing the HPV vaccine: researchers confront complex interplay of factors. J Natl Cancer Inst. 2009; 101(7):444-5. [PubMed: 19318636]

91. Kondo K, Ochi H, Matsumoto T, et al. Modification of human papillomavirus-like particle vaccine by insertion of the cross-reactive L2-epitopes. J Med Virol. 2008; 80(5):841-6. [PubMed: 18360909]

92. Kanda T, Kondo K. Development of an HPV vaccine for a broad spectrum of high-risk types. Hum Vaccin. 2009 Jan-Feb;5(1):43-5. [PubMed: 18690012]

93•. Schellenbacher C, Roden R, Kirnbauer R. Chimeric L1-L2 virus-like particles as potential broadspectrum human papillomavirus vaccines. J Virol. 2009; 83(19):10085-95. A study of the generation of a chimera virus-like particle presenting L2 on its surface for the induction of broadspectrum neutralizing antibodies against HPV. [PubMed: 19640991]

94. Lin J, Xu J, Albers AE, et al. New developments in therapeutic HPV vaccines. Curr Obstet Gynecol Rep. 2012; 1(3):106-15.

95. Lin K, Roosinovich E, Ma B, et al. Therapeutic HPV DNA vaccines. Immunol Res. 2010; 47(1-3): 86-112. [PubMed: 20066511]

96. Souders NC, Sewell DA, Pan ZK, et al. Listeria-based vaccines can overcome tolerance by expanding low avidity CD8 $+\mathrm{T}$ cells capable of eradicating a solid tumor in a transgenic mouse model of cancer. Cancer Immun. 2007; 7:2. [PubMed: 17279610]

97. Sewell DA, Pan ZK, Paterson Y. Listeria-based HPV-16 E7 vaccines limit autochthonous tumor growth in a transgenic mouse model for HPV-16 transformed tumors. Vaccine. 2008; 26(41): 5315-20. [PubMed: 18680778]

98. Adachi K, Kawana K, Yokoyama T, et al. Oral immunization with a Lactobacillus casei vaccine expressing human papillomavirus (HPV) type $16 \mathrm{E} 7$ is an effective strategy to induce mucosal cytotoxic lymphocytes against HPV16 E7. Vaccine. 2010; 28(16):2810-17. [PubMed: 20170766]

99. Quistian-Martinez D, Villatoro-Hernandez J, Loera-Arias MJ, et al. Efficient secretion of a modified E7 protein from human papilloma virus type-16 by Lactococcus lactis. Lett Appl Microbiol. 2010; 51(4):383-7. [PubMed: 20723044]

100•. Maciag PC, Radulovic S, Rothman J. The first clinical use of a live-attenuated Listeria monocytogenes vaccine: a Phase I safety study of Lm-LLO-E7 in patients with advanced carcinoma of the cervix. Vaccine. 2009; 27(30):3975-83. The first clinical study employing Listeria monocytogenes for the safe control of advanced cervical carcinoma. [PubMed: 19389451]

101. Radulovic S, Brankovic-Magic M, Malisic E, et al. Therapeutic cancer vaccines in cervical cancer: phase I study of Lovaxin-C. J BUON. 2009; 14(Suppl 1):S165-8. [PubMed: 19785060] 
102. Advaxis. Patients With HPV-16 +ve Oropharyngeal Carcinoma. 2012. REALISTIC: A Phase I, Dose Escalation Trial Of Recombinant Listeria Monocytogenes (Lm)-Based Vaccine Encoding Human Papilloma Virus Genotype 16 Target Antigens (ADXS11-001).

103. NCI. A Phase II Evaluation of ADXS11-001 (NSC 752718, BB-IND\#13,712) in the Treatment of Persistent or Recurrent Squamous or Non-Squamous Cell Carcinoma of the Cervix. 2012.

104. Advaxis. A Randomized, Single Blind, Placebo Controlled Phase 2 Study to Assess the Safety of ADXS11-001 for the Treatment of Cervical Intraepithelial Neoplasia Grade 2/3. 2012.

105. Wallecha A, French C, Petit R, et al. Lm-LLO-based immunotherapies and HPV-associated disease. J Oncol. 2012; 2012:542851. [PubMed: 22481930]

106. Lee DW, Anderson ME, Wu S, et al. Development of an adenoviral vaccine against E6 and E7 oncoproteins to prevent growth of human papillomavirus-positive cancer. Arch Otolaryngol Head Neck Surg. 2008; 134(12):1316-23. [PubMed: 19075129]

107. Gomez-Gutierrez JG, Elpek KG, Montes de Oca-Luna R, et al. Vaccination with an adenoviral vector expressing calreticulin-human papillomavirus 16 E7 fusion protein eradicates E7 expressing established tumors in mice. Cancer Immunol Immunother. 2007; 56(7):997-1007. [PubMed: 17146630]

108. Daemen T, Riezebos-Brilman A, Regts J, et al. Superior therapeutic efficacy of alphavirusmediated immunization against human papilloma virus type 16 antigens in a murine tumour model: effects of the route of immunization. Antivir Ther. 2004; 9(5):733-42. [PubMed: 15535411]

109. Cassetti MC, McElhiney SP, Shahabi V, et al. Antitumor efficacy of venezuelan equine encephalitis virus replicon particles encoding mutated HPV16 E6 and E7 genes. Vaccine. 2004; 22(3-4):520-7. [PubMed: 14670335]

110. Velders MP, McElhiney S, Cassetti MC, et al. Eradication of established tumors by vaccination with venezuelan equine encephalitis virus replicon particles delivering human papillomavirus 16 E7 RNA. Cancer Res. 2001; 61(21):7861-7. [PubMed: 11691804]

111. Hibbitts S. TA-CIN, a vaccine incorporating a recombinant HPV fusion protein (HPV16 L2E6E7) for the potential treatment of HPV16-associated genital diseases. Curr Opin Mol Ther. 2010; 12(5):598-606. [PubMed: 20886392]

112. Zurkova K, Babiarova K, Hainz P, et al. The expression of the soluble isoform of hFlt3 ligand by recombinant vaccinia virus enhances immunogenicity of the vector. Oncol Rep. 2009; 21(5): 1335-43. [PubMed: 19360312]

113. Hsieh CJ, Kim TW, Hung CF, et al. Enhancement of vaccinia vaccine potency by linkage of tumor antigen gene to gene encoding calreticulin. Vaccine. 2004; 22(29-30):3993-4001. [PubMed: 15364449]

114. Lamikanra A, Pan ZK, Isaacs SN, et al. Regression of established human papillomavirus type 16 (HPV-16) immortalized tumors in vivo by vaccinia viruses expressing different forms of HPV-16 E7 correlates with enhanced CD8(+) T-cell responses that home to the tumor site. J Virol. 2001; 75(20):9654-64. [PubMed: 11559797]

115. Kaufmann AM, Stern PL, Rankin EM, et al. Safety and immunogenicity of TA-HPV, a recombinant vaccinia virus expressing modified human papillomavirus (HPV)-16 and HPV-18 E6 and E7 genes, in women with progressive cervical cancer. Clin Cancer Res. 2002; 8(12): 3676-85. [PubMed: 12473576]

116. Borysiewicz LK, Fiander A, Nimako M, et al. A recombinant vaccinia virus encoding human papillomavirus types 16 and 18, E6 and E7 proteins as immunotherapy for cervical cancer. Lancet. 1996; 347(9014):1523-7. [PubMed: 8684105]

117. Davidson EJ, Boswell CM, Sehr P, et al. Immunological and clinical responses in women with vulval intraepithelial neoplasia vaccinated with a vaccinia virus encoding human papillomavirus 16/ 18 oncoproteins. Cancer Res. 2003; 63(18):6032-41. [PubMed: 14522932]

118. Baldwin PJ, van der Burg SH, Boswell CM, et al. Vaccinia-expressed human papillomavirus 16 and 18 e 6 and $\mathrm{e} 7$ as a therapeutic vaccination for vulval and vaginal intraepithelial neoplasia. Clin Cancer Res. 2003; 9(14):5205-13. [PubMed: 14614000]

119. Adams M, Borysiewicz L, Fiander A, et al. Clinical studies of human papilloma vaccines in preinvasive and invasive cancer. Vaccine. 2001; 19(17-19):2549-56. [PubMed: 11257391] 
120. Cancer EOfRaTo. ClinicalTrialsgov [Internet]. Bethesda (MD): National Library of Medicine (US); 1999. A phase II trial in patients with early cervical cancer to study the safety and the immunological effects of vaccination with TA-HPV, a live recombinant vaccinia virus expressing the human papilloma virus 16 and $18 \mathrm{E} 6$ and E7 proteins.

121. Corona Gutierrez CM, Tinoco A, Navarro T, et al. Therapeutic vaccination with MVA E2 can eliminate precancerous lesions (CIN 1, CIN 2, and CIN 3) associated with infection by oncogenic human papillomavirus. Hum Gene Ther. 2004; 15(5):421-31. [PubMed: 15144573]

122. Garcia-Hernandez E, Gonzalez-Sanchez JL, Andrade-Manzano A, et al. Regression of papilloma high-grade lesions (CIN 2 and CIN 3) is stimulated by therapeutic vaccination with MVA E2 recombinant vaccine. Cancer Gene Ther. 2006; 13(6):592-7. [PubMed: 16456551]

123. Albarran YCA, de la Garza A, Cruz Quiroz BJ, et al. MVA E2 recombinant vaccine in the treatment of human papillomavirus infection in men presenting intraurethral flat condyloma: a phase I/II study. BioDrugs. 2007; 21(1):47-59. [PubMed: 17263589]

124. Transgene. Transgene Reports Randomized Phase 2b Data with its Therapeutic HPV Vaccine TG4001 in Women with CIN2/3 Intraepithelial Cervical Neoplasia. 2012. Available from: http:// www.transgene.fr/index.php?option=com_press_release\&task=download\&id=208\&l=en [Cited 20 July 2012]

125. Fiander AN, Tristram AJ, Davidson EJ, et al. Prime-boost vaccination strategy in women with high-grade, noncervical anogenital intraepithelial neoplasia: clinical results from a multicenter phase II trial. Int J Gynecol Cancer. 2006 May-Jun;16(3):1075-81. [PubMed: 16803488]

126. University JH. ClinicalTrials.gov [Internet]. National Library of Medicine (US); Bethesda (MD): 2008. Vaccine therapy with or without imiquimod in treating patients with grade 3 cervical intraepithelial neoplasia.

127. Qin Y, Wang XH, Cui HL, et al. Human papillomavirus type 16 E7 peptide(38-61) linked with an immunoglobulin $\mathrm{G}$ fragment provides protective immunity in mice. Gynecol Oncol. 2005; 96(2):475-83. [PubMed: 15661238]

128. Sharma RK, Elpek KG, Yolcu ES, et al. Costimulation as a platform for the development of vaccines: a peptide-based vaccine containing a novel form of 4-1BB ligand eradicates established tumors. Cancer Res. 2009; 69(10):4319-26. [PubMed: 19435920]

129. Yan W, Chen WC, Liu Z, et al. Bryostatin-I: a dendritic cell stimulator for chemokines induction and a promising adjuvant for a peptide based cancer vaccine. Cytokine. 2010; 52(3):238-44. [PubMed: 20869878]

130. Zwaveling S, Ferreira Mota SC, Nouta J, et al. Established human papillomavirus type 16expressing tumors are effectively eradicated following vaccination with long peptides. $\mathrm{J}$ Immunol. 2002; 169(1):350-8. [PubMed: 12077264]

131. Daftarian P, Mansour M, Benoit AC, et al. Eradication of established HPV 16-expressing tumors by a single administration of a vaccine composed of a liposome-encapsulated CTL-T helper fusion peptide in a water-in-oil emulsion. Vaccine. 2006; 24(24):5235-44. [PubMed: 16675074]

132. Zhang YQ, Tsai YC, Monie A, et al. Carrageenan as an adjuvant to enhance peptide-based vaccine potency. Vaccine. 2010; 28(32):5212-19. [PubMed: 20541583]

133. Wu CY, Yang HY, Monie A, et al. Intraperitoneal administration of poly(I: C) with polyethylenimine leads to significant antitumor immunity against murine ovarian tumors. Cancer Immunol Immunother. 2011; 60(8):1085-96. [PubMed: 21526359]

134. Barrios K, Celis E. TriVax-HPV: an improved peptide-based therapeutic vaccination strategy against human papillomavirus-induced cancers. Cancer Immunol Immunother. 2012; 61(8): 1307-17. [PubMed: 22527249]

135. Steller MA, Gurski KJ, Murakami M, et al. Cell-mediated immunological responses in cervical and vaginal cancer patients immunized with a lipidated epitope of human papillomavirus type 16 E7. Clin Cancer Res. 1998; 4(9):2103-9. [PubMed: 9748126]

136. van Driel WJ, Ressing ME, Kenter GG, et al. Vaccination with HPV16 peptides of patients with advanced cervical carcinoma: clinical evaluation of a phase I-II trial. Eur J Cancer. 1999; 35(6): 946-52. [PubMed: 10533477] 
137. Ressing ME, van Driel WJ, Brandt RM, et al. Detection of T helper responses, but not of human papillomavirus-specific cytotoxic $\mathrm{T}$ lymphocyte responses, after peptide vaccination of patients with cervical carcinoma. J Immunother. 2000 Mar-Apr;23(2):255-66. [PubMed: 10746552]

138. Muderspach L, Wilczynski S, Roman L, et al. A phase I trial of a human papillomavirus (HPV) peptide vaccine for women with high-grade cervical and vulvar intraepithelial neoplasia who are HPV 16 positive. Clin Cancer Res. 2000; 6(9):3406-16. [PubMed: 10999722]

139. Kenter GG, Welters MJ, Valentijn AR, et al. Phase I immunotherapeutic trial with long peptides spanning the E6 and E7 sequences of high-risk human papillomavirus 16 in end-stage cervical cancer patients shows low toxicity and robust immunogenicity. Clin Cancer Res. 2008; 14(1): 169-77. [PubMed: 18172268]

140. Welters MJ, Kenter GG, Piersma SJ, et al. Induction of tumor-specific CD4+ and CD8+ T-cell immunity in cervical cancer patients by a human papillomavirus type $16 \mathrm{E} 6$ and E7 long peptides vaccine. Clin Cancer Res. 2008; 14(1):178-87. [PubMed: 18172269]

141••. Kenter GG, Welters MJ, Valentijn AR, et al. Vaccination against HPV-16 oncoproteins for vulvar intraepithelial neoplasia. N Engl J Med. 2009; 361(19):1838-47. An important clinical trial indicating the efficacy of a long-peptide vaccine against HPV 16 E6 and E7 oncoproteins in generating clinical responses in women with HPV 16-associated vulvar intraepithelial neoplasia. [PubMed: 19890126]

142. Welters MJ, Kenter GG, de Vos van Steenwijk PJ, et al. Success or failure of vaccination for HPV16-positive vulvar lesions correlates with kinetics and phenotype of induced T-cell responses. Proc Natl Acad Sci USA. 2010; 107(26):11895-9. [PubMed: 20547850]

143. de Vos van Steenwijk PJ, Ramwadhdoebe TH, Lowik MJ, et al. A placebo-controlled randomized HPV16 synthetic long-peptide vaccination study in women with high-grade cervical squamous intraepithelial lesions. Cancer Immunol Immunother. 2012; 61(9):1485-92. [PubMed: 22684521]

144. Cui Z, Huang L. Liposome-polycation-DNA (LPD) particle as a carrier and adjuvant for proteinbased vaccines: therapeutic effect against cervical cancer. Cancer Immunol Immunother. 2005; 54(12):1180-90. [PubMed: 15846491]

145. Stewart TJ, Drane D, Malliaros J, et al. ISCOMATRIX adjuvant: an adjuvant suitable for use in anticancer vaccines. Vaccine. 2004; 22(27-28):3738-43. [PubMed: 15315854]

146. Kang TH, Monie A, Wu LS, et al. Enhancement of protein vaccine potency by in vivo electroporation mediated intramuscular injection. Vaccine. 2011; 29(5):1082-9. [PubMed: 21130752]

147. Preville X, Ladant D, Timmerman B, et al. Eradication of established tumors by vaccination with recombinant Bordetella pertussis adenylate cyclase carrying the human papillomavirus 16 E7 oncoprotein. Cancer Res. 2005; 65(2):641-9. [PubMed: 15695409]

148. Chu NR, Wu HB, Wu T, et al. Immunotherapy of a human papillomavirus (HPV) type 16 E7expressing tumour by administration of fusion protein comprising Mycobacterium bovis bacille Calmette-Guerin (BCG) hsp65 and HPV16 E7. Clin Exp Immunol. 2000; 121(2):216-25. [PubMed: 10931134]

149. Liu H, Wu BH, Rowse GJ, et al. Induction of CD4-independent E7-specific CD8+ memory response by heat shock fusion protein. Clin Vaccine Immunol. 2007; 14(8):1013-23. [PubMed: 17596433]

150. Liao CW, Chen CA, Lee CN, et al. Fusion protein vaccine by domains of bacterial exotoxin linked with a tumor antigen generates potent immunologic responses and antitumor effects. Cancer Res. 2005; 65(19):9089-98. [PubMed: 16204084]

151. Granadillo M, Vallespi MG, Batte A, et al. A novel fusion protein-based vaccine comprising a cell penetrating and immunostimulatory peptide linked to human papillomavirus (HPV) type 16 E7 antigen generates potent immunologic and anti-tumor responses in mice. Vaccine. 2011; 29(5):920-30. [PubMed: 21145912]

152. Huang CY, Chen JJ, Shen KY, et al. Recombinant lipidated HPV E7 induces a Th-1-biased immune response and protective immunity against cervical cancer in a mouse model. PLoS ONE. 2012; 7(7):e40970. [PubMed: 22815882] 
153. Derkay CS, Smith RJ, McClay J, et al. HspE7 treatment of pediatric recurrent respiratory papillomatosis: final results of an open-label trial. Ann Otol Rhinol Laryngol. 2005; 114(9):730 7. [PubMed: 16240938]

154. Goldstone SE, Palefsky JM, Winnett MT, et al. Activity of HspE7, a novel immunotherapy, in patients with anogenital warts. Dis Colon Rectum. 2002; 45(4):502-7. [PubMed: 12006932]

155. Roman LD, Wilczynski S, Muderspach LI, et al. A phase II study of Hsp-7 (SGN-00101) in women with high-grade cervical intraepithelial neoplasia. Gynecol Oncol. 2007; 106(3):558-66. [PubMed: 17631950]

156. Einstein MH, Kadish AS, Burk RD, et al. Heat shock fusion protein-based immunotherapy for treatment of cervical intraepithelial neoplasia III. Gynecol Oncol. 2007; 106(3):453-60. [PubMed: 17586030]

157. Van Doorslaer K, Reimers LL, Studentsov YY, et al. Serological response to an HPV16 E7 based therapeutic vaccine in women with high-grade cervical dysplasia. Gynecol Oncol. 2010; 116(2): 208-12. [PubMed: 19555999]

158. Newswire, P. Nventa Announces Final Data from HspE7 Phase 1 Cervical Dysplasia Trial. PR Newswire United Business Media: PR Newswire Association LLC; 2008.

159. Center SKCC. ClinicalTrialsgov [Internet]. National Library of Medicine (US); Bethesda (MD): 2009. A pilot study of pnGVL4a-CRT/E7 (Detox) for the treatment of patients with HPV16+ cervical intraepithelial neoplasia 2/3 (CIN2/3).

160. Klencke B, Matijevic M, Urban RG, et al. Encapsulated plasmid DNA treatment for human papillomavirus 16-associated anal dysplasia: a Phase I study of ZYC101. Clin Cancer Res. 2002; 8(5):1028-37. [PubMed: 12006515]

161. Sheets EE, Urban RG, Crum CP, et al. Immunotherapy of human cervical high-grade cervical intraepithelial neoplasia with microparticle-delivered human papillomavirus $16 \mathrm{E} 7$ plasmid DNA. Am J Obstet Gynecol. 2003; 188(4):916-26. [PubMed: 12712086]

162. Garcia F, Petry KU, Muderspach L, et al. ZYC101a for treatment of high-grade cervical intraepithelial neoplasia: a randomized controlled trial. Obstet Gynecol. 2004; 103(2):317-26. [PubMed: 14754702]

163. Alvarez-Salas LM. Amolimogene bepiplasmid, a DNA-based therapeutic encoding the E6 and E7 epitopes from HPV, for cervical and anal dysplasia. Curr Opin Mol Ther. 2008; 10(6):622-8. [PubMed: 19051140]

164. Matijevic M, Hedley ML, Urban RG, et al. Immunization with a poly (lactide co-glycolide) encapsulated plasmid DNA expressing antigenic regions of HPV 16 and 18 results in an increase in the precursor frequency of T cells that respond to epitopes from HPV 16, 18, 6 and 11. Cell Immunol. 2011; 270(1):62-9. [PubMed: 21550027]

165. Bodles-Brakhop AM, Heller R, Draghia-Akli R. Electroporation for the delivery of DNA-based vaccines and immunotherapeutics: current clinical developments. Mol Ther. 2009; 17(4):585-92. [PubMed: 19223870]

166••. Bagarazzi ML, Yan J, Morrow MP, et al. Immunotherapy Against HPV16/ 18 Generates Potent TH1 and Cytotoxic Cellular Immune Responses. Sci Transl Med. 2012; 4(155):155ra38. An important Phase I clinical trial reporting the safety and efficacy of an HPV 16-18 vaccine delivered by in vivo electroporation.

167. Pharmaceuticals I. Phase II Placebo Controlled Study of VGX-3100, (HPV16 E6/E7, HPV18 E6/ E7 DNA Vaccine) Delivered IM Followed by Electroporation With Cellectra-5P for the Treatment of Biopsy-proven CIN 2/3 or CIN 3 With Documented HPV 16 or 18. 2012

168. Trimble CL, Peng S, Kos F, et al. A phase I trial of a human papillomavirus DNA vaccine for HPV16 + cervical intraepithelial neoplasia 2/3. Clin Cancer Res. 2009; 15(1):361-7. [PubMed: 19118066]

169. Chung $\mathrm{CH}$, Gillison ML. Human papillomavirus in head and neck cancer: its role in pathogenesis and clinical implications. Clin Cancer Res. 2009; 15(22):6758-62. [PubMed: 19861444]

170. Bilu D, Sauder DN. Imiquimod: modes of action. Br J Dermatol. 2003; 149(Suppl 66):5-8. [PubMed: 14616337]

171. Chuang CM, Monie A, Hung CF, et al. Treatment with imiquimod enhances antitumor immunity induced by therapeutic HPV DNA vaccination. J Biomed Sci. 2010; 17:32. [PubMed: 20426849] 
172. Santin AD, Bellone S, Palmieri M, et al. Human papillomavirus type 16 and 18 E7-pulsed dendritic cell vaccination of stage IB or IIA cervical cancer patients: a phase I escalating-dose trial. J Virol. 2008; 82(4):1968-79. [PubMed: 18057249]

173•. Tseng CW, Trimble C, Zeng Q, et al. Low-dose radiation enhances therapeutic HPV DNA vaccination in tumor-bearing hosts. Cancer Immunol Immunother. 2009; 58(5):737-48. An important experimental study suggesting that low-dose radiation enhanced the efficacy of therapeutic HPV DNA vaccination in E7-expressing tumor-bearing mice. [PubMed: 18815785]

174. Kang TH, Lee JH, Song CK, et al. Epigallocatechin-3-gallate enhances CD8 + T cell-mediated antitumor immunity induced by DNA vaccination. Cancer Res. 2007; 67(2):802-11. [PubMed: 17234792]

175•. Tseng CW, Hung CF, Alvarez RD, et al. Pretreatment with cisplatin enhances E7-specific CD8+ T-Cell-mediated antitumor immunity induced by DNA vaccination. Clin Cancer Res. 2008; 14(10):3185-92. An important study indicating that chemotherapy with cisplatin enhanced the potency of subsequent administration of an HPV DNA vaccine. [PubMed: 18483387]

176. Tseng CW, Monie A, Wu CY, et al. Treatment with proteasome inhibitor bortezomib enhances antigen-specific CD8+ T-cell-mediated antitumor immunity induced by DNA vaccination. J Mol Med (Berl). 2008; 86(8):899-908. [PubMed: 18542898]

177. Zeng Q, Peng S, Monie A, et al. Control of cervicovaginal HPV-16 E7-expressing tumors by the combination of therapeutic HPV vaccination and vascular disrupting agents. Hum Gene Ther. 2011; 22(7):809-19. [PubMed: 21128743]

178. Peng S, Monie A, Pang X, et al. Vascular disrupting agent DMXAA enhances the antitumor effects generated by therapeutic HPV DNA vaccines. J Biomed Sci. 2011; 18:21. [PubMed: 21385449]

179. Chuang CM, Monie A, Wu A, et al. Combination of apigenin treatment with therapeutic HPV DNA vaccination generates enhanced therapeutic antitumor effects. J Biomed Sci. 2009; 16:49. [PubMed: 19473507]

180. Transgene. Transgene and Roche Modify the Clinical Development Programme for their HPVtargeted Immunotherapy TG4001/R3484. 2008. [cited 2012 July 20]; Available from: http:// www.transgene.fr/index.php?option=com_press_release\&task=download\&id=22\&l=en

181. Frazer IH, Quinn M, Nicklin JL, et al. Phase 1 study of HPV16-specific immunotherapy with E6E7 fusion protein and ISCOMATRIX adjuvant in women with cervical intraepithelial neoplasia. Vaccine. 2004 Nov 25; 23(2):172-81. [PubMed: 15531034]

182. Anderson JS, Hoy J, Hillman R, et al. A randomized, placebo-controlled, dose-escalation study to determine the safety, tolerability, and immunogenicity of an HPV-16 therapeutic vaccine in HIVpositive participants with oncogenic HPV infection of the anus. J Acquir Immune Defic Syndr. 2009 Nov 1; 52(3):371-81. [PubMed: 19661810]

183. Hallez S, Simon P, Maudoux F, et al. Phase I/II trial of immunogenicity of a human papillomavirus (HPV) type $16 \mathrm{E} 7$ protein-based vaccine in women with oncogenic HPV-positive cervical intraepithelial neoplasia. Cancer immunology, immunotherapy: CII. 2004 Jul; 53(7): 642-50.

184. Palefsky JM, Berry JM, Jay N, et al. A trial of SGN-00101 (HspE7) to treat high-grade anal intraepithelial neoplasia in HIV-positive individuals. AIDS. 2006; 20(8):1151-5. [PubMed: 16691066]

185. Center, SKCC. Clinical Trials.gov [Internet]. Bethesda (MD): National Library of Medicine (US); 2005. A Phase I/II Clinical Trial of pNGVL4a-Sig/E7 (Detox)/HSP70 for the Treatment of Patients With HPV 16+ Cervical Intraepithelial Neoplasia 2/3 (CIN2/3).

186. Gillison. personal communication.

187. Pharmaceuticals, I. ClinicalTrials.gov [Internet]. Bethesda (MD): National Library of Medicine (US); Phase I Open Label, Dose Escalation Study to Evaluate the Safety, Tolerability and Immunogenicity of Human Papillomavirus (HPV) DNA Plasmid (VGX-3100) + Electroporation (EP) in Adult Females Post Surgical or Ablative Treatment of Grade 2 or 3 Cervical Intraepithelial Neoplasia (CIN).

188. Pharmaceuticals, I. ClinicalTrials.gov [Internet]. Bethesda (MD): National Library of Medicine (US); 2010. Phase I, Open-label Study to Evaluate the Safety, Tolerability and Immunogenicity 
of a Fourth Dose of Human Papillomavirus (HPV) DNA Plasmid (VGX-3100) + Electroporation (EP) in Adult Females Previously Immunized With VGX-3100.

189. ClinicalTrials.gov [Internet]. Bethesda (MD): National Library of Medicine (US); 2005. Hospital, NTU A Pilot Study for the Immunotherapy of Recurrent Cervical Cancers Using Dendritic Cells (DCs) Pulsed With Human Papillomavirus Type 16 E7 Antigen.

190. Smyth LJ, Van Poelgeest MI, Davidson EJ, et al. Immunological responses in women with human papillomavirus type 16 (HPV-16)-associated anogenital intraepithelial neoplasia induced by heterologous prime-boost HPV-16 oncogene vaccination. Clin Cancer Res. 2004; 10:2954-2961. [PubMed: 15131030]

191. Davidson EJ, Faulkner RL, Sehr P, et al. Effect of TA-CIN (HPV 16 L2E6E7) booster immunization in vulval intraepithelial neoplasia patients previously vaccinated with TA-HPV (vaccinia virus encoding HPV 16/18 E6E7). Vaccine. 2004; 22:2722-2729. [PubMed: 15246603] 


\section{Article highlights}

- Significant steps toward the ideal prophylactic and therapeutic HPV vaccines have been made.

- The current commercial preventive HPV vaccines have been shown to be efficacious in generating capsid-specific neutralizing antibody responses to HPV-16 and HPV-18, the two most common types associated with cervical cancer.

- Next-generation preventive HPV vaccine candidates have explored the issues of lowering cost, increasing duration of efficacy, and broadening protection through multivalent virus-like particle (VLP) vaccines, L1 capsomer vaccines, L2 capsid protein-targeted vaccines, and chimeric L1/L2 VLP vaccines.

- Antigen-specific immunotherapy generated by therapeutic HPV vaccines has focused on enhancing T cell-mediated killing of HPV-transformed tumor cells, which constitutively express HPV-encoded proteins, E6 and E7.

- Therapeutic HPV vaccines targeting HPV E6 and E7 have been explored in clinical trials using live vector, peptide, protein, DNA, RNA replicon, and dendritic cell-based HPV vaccines.

- The progress of therapeutic HPV vaccines through clinical trials may likely require combination strategies of different therapeutic modalities.

This box summarizes key points contained in the article. 


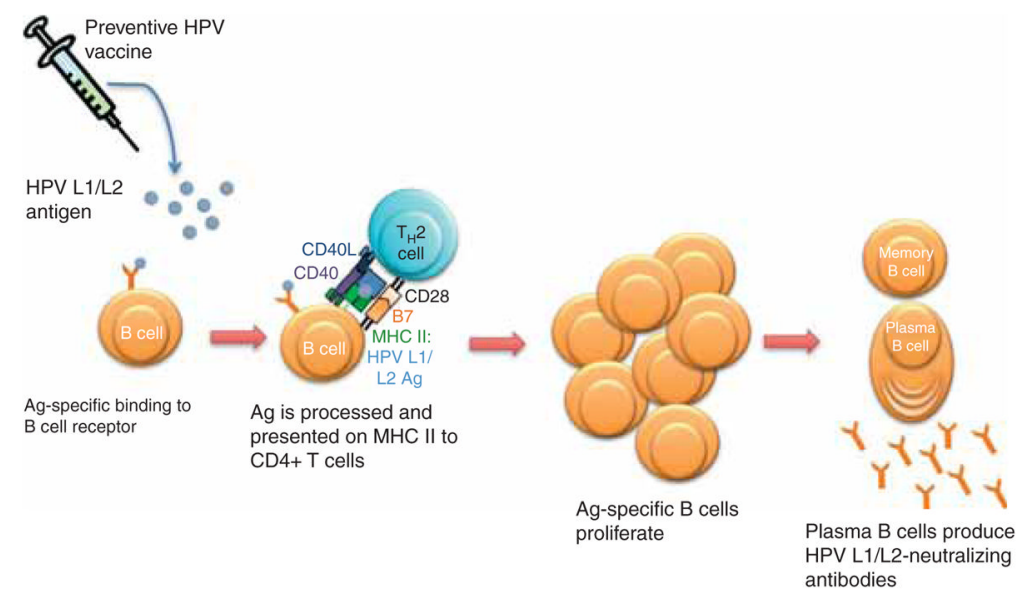

Figure 1. Preventive HPV vaccination focuses on generating neutralizing antibodies for prevention of infection

Humoral immunity involves the interaction between an HPV capsid antigen (L1 and/or L2) and an antigen-specific B-cell receptor. The B cell internalizes the antigen, processes it, and presents it on an MHC class II molecule. The MHC II: L1/L2 antigen complex is recognized by a specific $\mathrm{T}_{\mathrm{H}} 2$-cell receptor. This $\mathrm{T}_{\mathrm{H}} 2$ cell has been activated by a dendritic cell presenting the L1/L2 antigen, causing it to mature and specifically recognize the MHC II: $\mathrm{L} 1 / \mathrm{L} 2$ complex. The $\mathrm{T}_{\mathrm{H}} 2$ cell activates the $\mathrm{B}$ cell to proliferate and differentiate into memory B cells and plasma B cells, which produce HPV-specific antibodies. The HPV antigen-specific antibodies prevent HPV infection by binding the viruses and thus preventing their entry into host cells. 


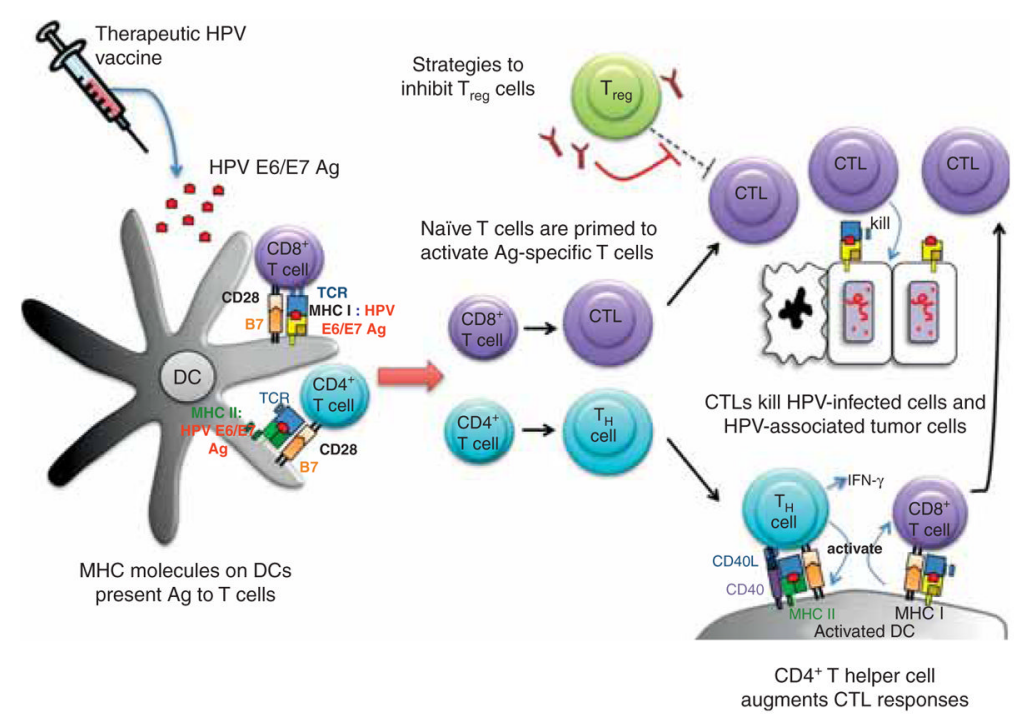

Figure 2. Therapeutic HPV vaccination focuses on generating cell-mediated immunity for clearance of infection

Cell-mediated immunity involves the interaction between professional antigen-presenting cells (particularly dendritic cells) and T cells. Dendritic cells present the MHC:peptide complex to prime naïve T cells to become effector T cells. Effector T cells mediate therapeutic effects; effector CD8+ T cells, also known as cytotoxic T lymphocytes (CTL), mediate antigen-specific killing of tumor cells, and effector CD4+ T cells differentiate into T helper $\left(\mathrm{T}_{\mathrm{H}}\right)$ cells to augment CTL immune responses through the production of IFN- $\mathrm{\gamma}$. Regulatory $\mathrm{T}$ cells $\left(\mathrm{T}_{\mathrm{reg}}\right.$ ) regulate immune responses by suppressing CTL and $\mathrm{T}_{\mathrm{H}}$ cells. Strategies to inhibit the Foxp3+ CD4+ CD25+ Treg cells, such as the use of anti-CD25 monoclonal antibody, may improve CTL immune responses. 


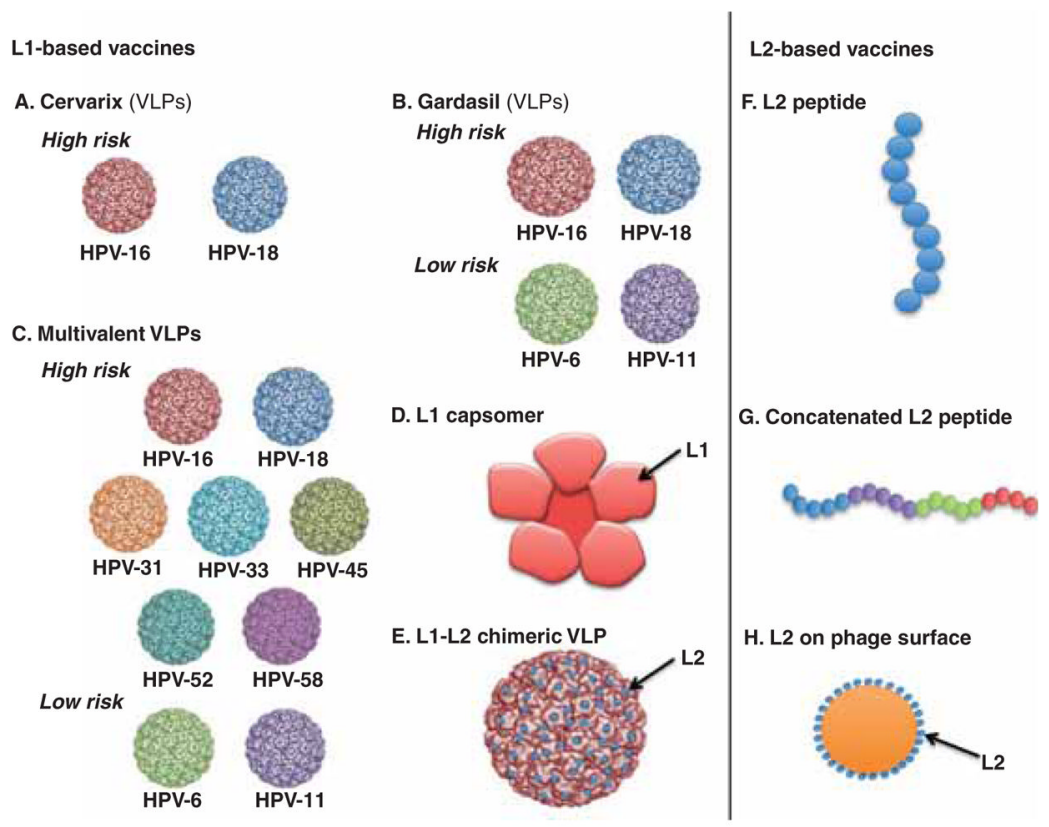

Figure 3. Schematic diagram to depict the next generation of preventive HPV vaccines based on HPV capsid proteins ( $L 1$ and/or L2)

A. Cervarix composed of HPV-16 and HPV-18 VLPs. B. Gardasil composed of HPV-6, HPV-11, HPV-16 and HPV-18 VLPs. C. Multivalent VLP vaccines composed of HPV-6, HPV-11, HPV-16, HPV-18, HPV-31, HPV-33, HPV-45, HPV-52 and HPV-58 VLPs (V503 Merck). D. L1 capsomer vaccine. E. Chimeric L1-L2 VLP vaccine with L2 on the surface. F. L2 peptide vaccine. G. Concatenated L2 peptide vaccine. H. L2 peptides displayed on the surface of bacteriophage VLP. 
Table 1

Overview of Commercial Preventive HPV Vaccines.

\begin{tabular}{|c|c|c|}
\hline & Gardasil & Cervarix \\
\hline Manufacturer & Merck & GlaxoSmithKline \\
\hline HPV types & $16,18,6,11$ & 16,18 \\
\hline Amount of HPV L1 protein & $\begin{array}{l}20 \mu \mathrm{g} \mathrm{HPV}-6 \\
40 \mu \mathrm{g} \mathrm{HPV}-11 \\
40 \mu \mathrm{g} \mathrm{HPV}-16 \\
20 \mu \mathrm{g} \mathrm{HPV}-18\end{array}$ & $\begin{array}{l}20 \mu \mathrm{g} \mathrm{HPV}-16 \\
20 \mu \mathrm{g} \mathrm{HPV}-18\end{array}$ \\
\hline Adjuvant & $225 \mu \mathrm{g}$ aluminum hydroxyphosphate sulfate & $\begin{array}{l}\text { AS } 04 \text { composed of: } 500 \mu g \text { aluminum hydroxide } \\
50 \mu g \text { MPL (3-O-desacyl-4'-monophosphoryl } \\
\text { lipid A) }\end{array}$ \\
\hline FDA Approval in US & June 2006 & October 2009 \\
\hline Regimen & 3 doses: Months 0, 2, 6 & 3 doses: Months 0, 1, 6 \\
\hline Cost & $\$ 120$ USD per dose & $\$ 100$ USD per dose \\
\hline Antigen Source & Saccharomyces cerevisiae (bread yeast), expressing L1 & $\begin{array}{l}\text { Trichoplusia ni insect cell line infected with L1 } \\
\text { encoding recombinant baculovirus }\end{array}$ \\
\hline Indications & $\begin{array}{l}\text { Girls and young women: age } 9-26 \\
\text { For the prevention of cervical, vulvar, vaginal cancer, and } \\
\text { anal cancer and associated pre-cancerous lesions and } \\
\text { genital warts } \\
\text { Boys and young men: ages } 9-26 \\
\text { For the prevention of anal cancer and associated pre- } \\
\text { cancerous lesions, and genital warts }\end{array}$ & $\begin{array}{l}\text { Girls and young women: age } 10-25 \\
\text { For the prevention of cervical cancer and } \\
\text { associated pre-cancerous lesions }\end{array}$ \\
\hline Duration of protection & At least 6.4 years $[60,61]$ & At least 8.4 years $[40,63]$ \\
\hline Antibody titers & \multicolumn{2}{|c|}{$\begin{array}{l}\text { HPV-16: } 2.3-4.8 \text { times higher for Cervarix compared with Gardasil, across all age groups } \\
\text { HPV-18: } 6.8-9.1 \text { times higher with Cervarix [64] }\end{array}$} \\
\hline
\end{tabular}



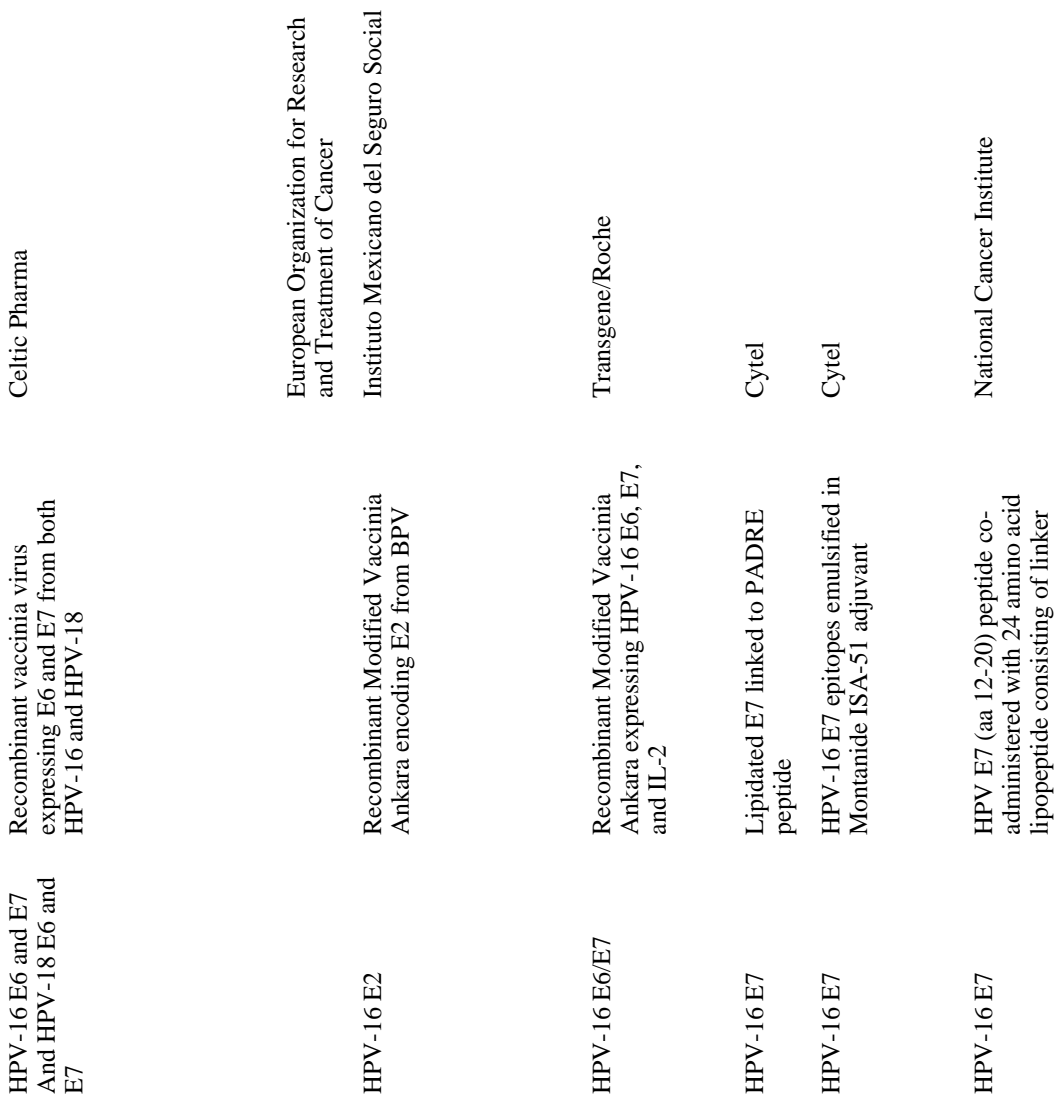


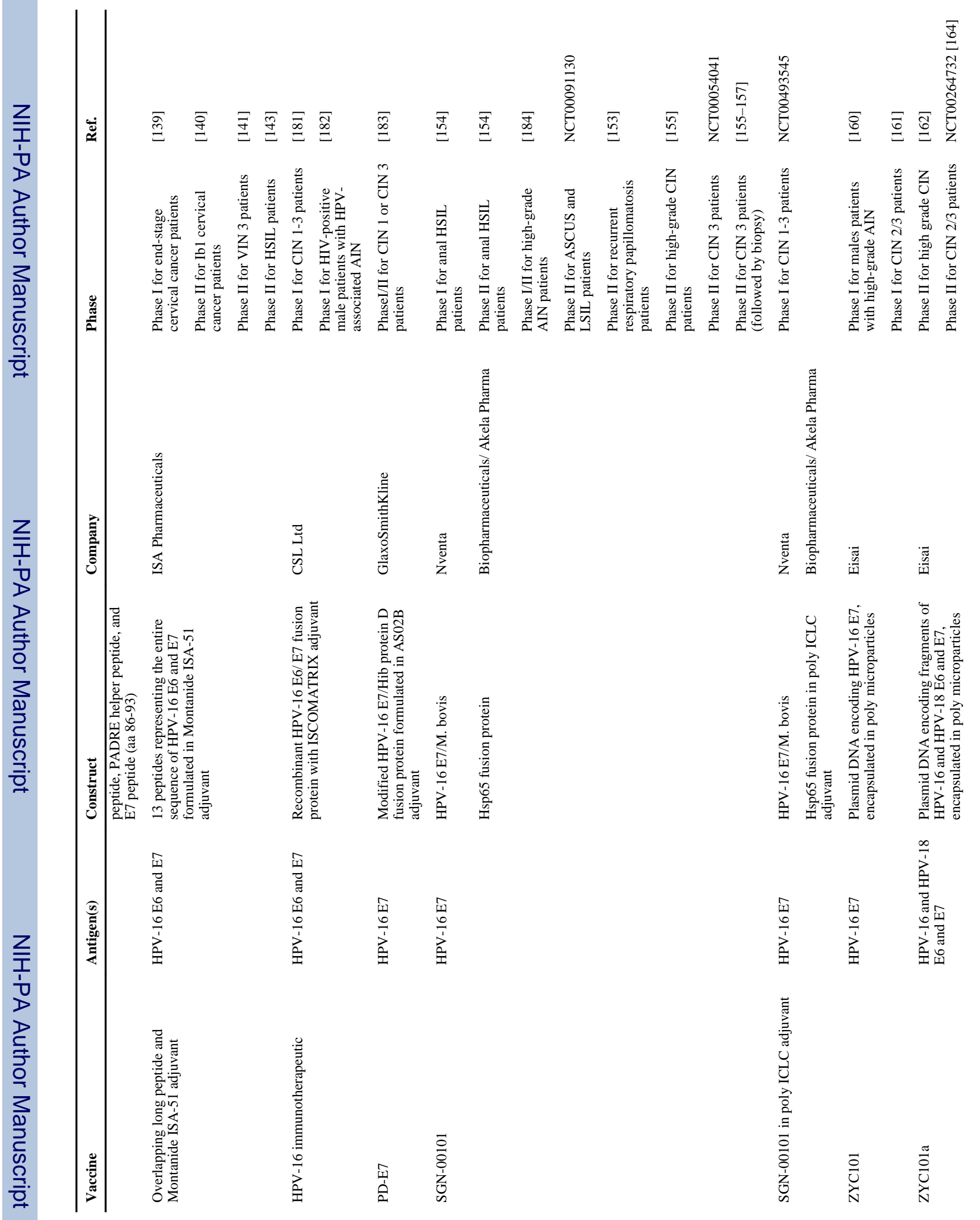




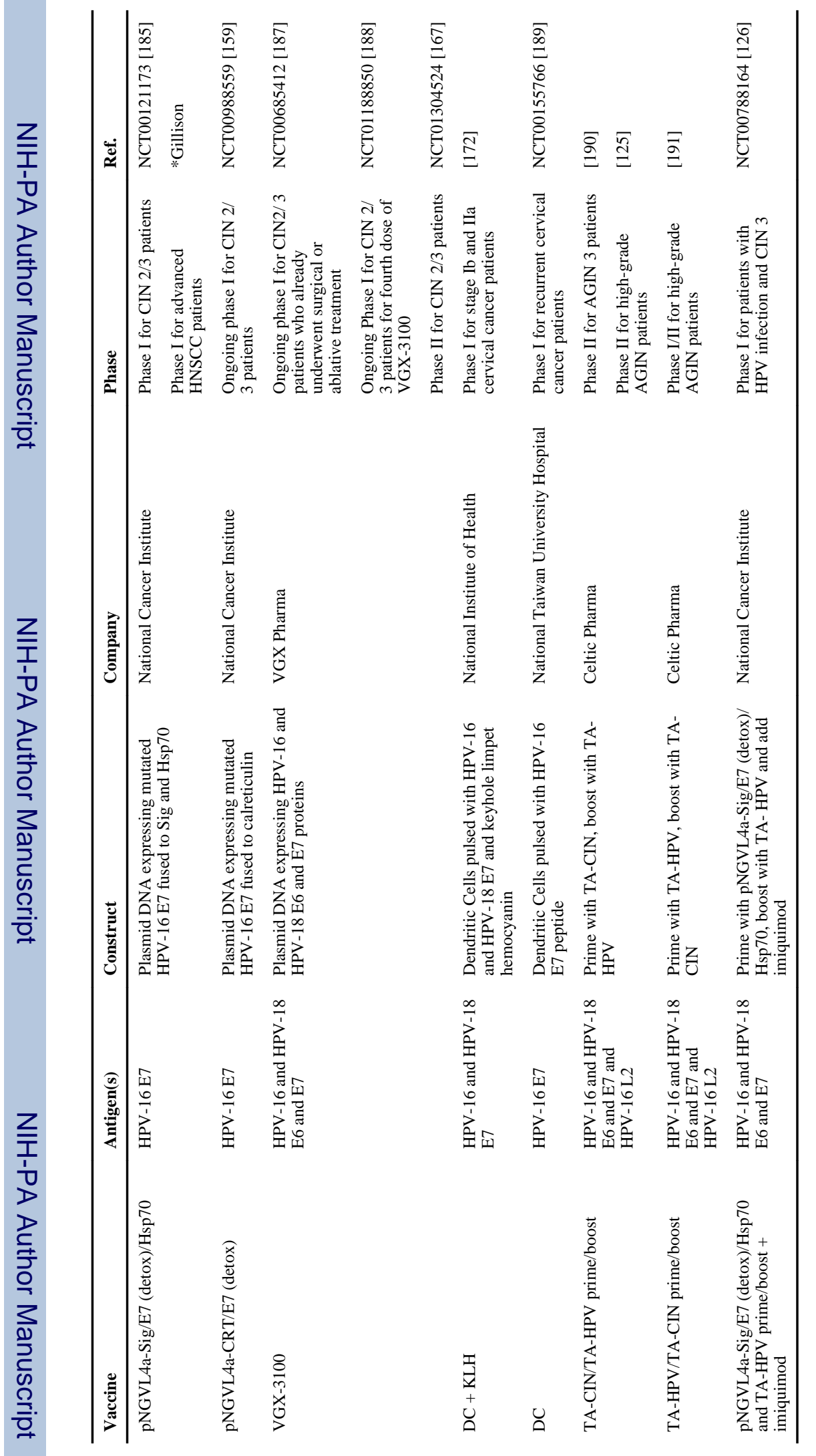

